Portland State University

PDXScholar

3-12-1971

\title{
Die Prostituierte in Frank Wedekinds Dramen
}

Philip A. Mellen

Portland State University

Follow this and additional works at: https://pdxscholar.library.pdx.edu/open_access_etds

Part of the German Literature Commons Let us know how access to this document benefits you.

\section{Recommended Citation}

Mellen, Philip A., "Die Prostituierte in Frank Wedekinds Dramen" (1971). Dissertations and Theses. Paper 715.

https://doi.org/10.15760/etd.715

This Thesis is brought to you for free and open access. It has been accepted for inclusion in Dissertations and Theses by an authorized administrator of PDXScholar. Please contact us if we can make this document more accessible: pdxscholar@pdx.edu. 
AN ABSTRACT OF THE THESIS OF Philip A. Mellen for the Master of Arts in German presented Maroh 12, 1971.

Title: Die Prostituierte in Frank Wedekinds Dramen. (The Prostitute in Frank Tedekind's Dramas.)

APPROVED BY THE MEMBERS OF THE THESIS COMMITTEE:
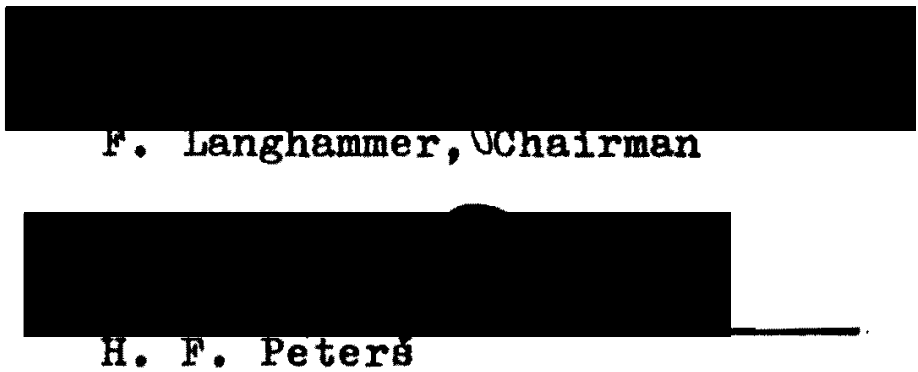

The prostitute and the concept of prostitution played a meaningful role in both Frank Fedekind's 11 fe and hio dramatic efforts. From his early youth to the writing of his drama Schloss Wetterstein (1910), Wedekind remained deepiy interested in the personal, social and philosophical problems generated by the existence of the prostitute and what he imagined her sensually based philosophy of life to be. Four of Wedekind's dramas were dealt with, which seem to be representative of his struggle to vindicate his own corresponding philosophy of sensuality.

The first drama, Eling Erweckung (Elin's Awakening-1887), is important as his first drama dealing with the prostitute. It 18 largely socio-critical in tone and develops character types, which will later appear in other Fedekind dramas concerning 
the prostitute. Das Sonnenspektrum (Spectrum of the Sun-1894), the fragmentary, second play analysed, develops the theme of sensual foy carried to its practical 1imit: a garden of physical love, where art and man's physical appetites live in harmony. The philosophical implications of unrestrained phy" sical love, as embodied by the prostitute, take on a darker hue in the third drama, Tod und Teufel (Death and the Devil1905). In this drama Wedekind's disillusionment with sensual love is shown. Its characters are not freely enjoying their unrestrained sensuality; they are driven by Inner, bestial forces to their destruction. Wedekind attempts to rescue daemonic sexuality in the final drama worked with, Schloss Wetterstein (Wetterstein Castle). He creates the "Edelhure" (Noble Whore) in this work, who triumphs philosophically over sensual pleasure, but pays with her life. Her death is proud, but real. With Schloss Wetterstein ends Wedekind's attempt to reconcile unrestrained sensuality with practical reality. He found that the prostitute could not outrun the fate inevitably awaiting her, if she (and himself) looked for the meaning of life on the dark side of Man's existence. Death only, awaits those who open Pandora's Box. 
DIE PROSTITUIERTE IN FRANK WEDEKIINDS DRAUEN

PHIIIP A. MELCERT

A thesis oubmitted in partial fulfillment of the requiremente for the degree of

MASTER OF ARTS

in

GRRMAII

Portland State Unirersity

1971 
TO THE OFFICE OF GRADUATE STUDIES:

The members of the Committee approve the thesis of Ph1lip A. Mellen presented March 12, 1971.

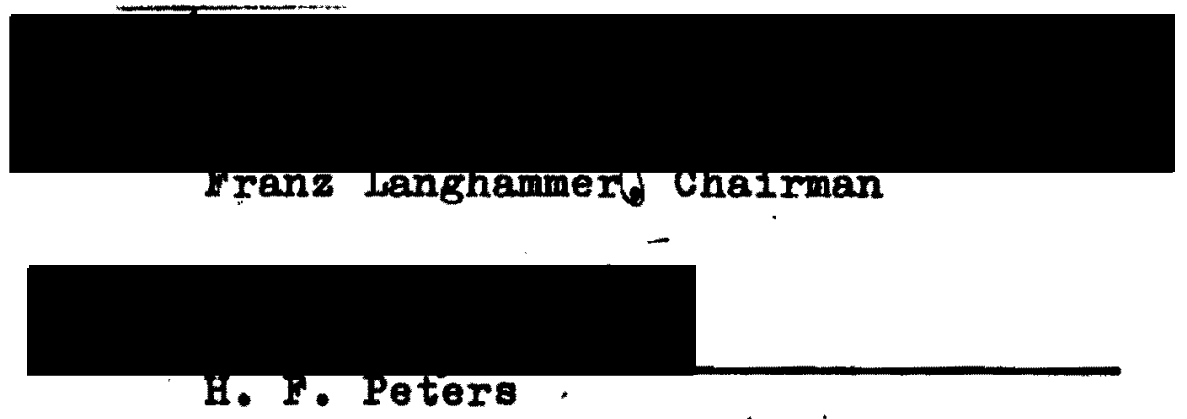

\section{APPROVED:}

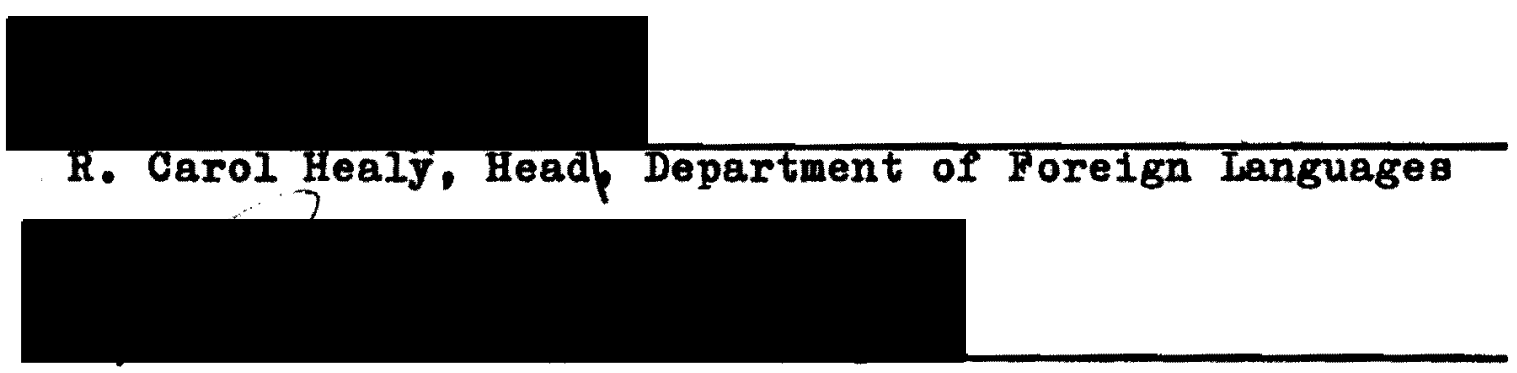

Darid T. Clark, Dean of Graduate Studies

March 15, 1971 


\section{INHALTSVERZEICHNIS}

SEITE

RAPITEL

I EINLEITUNG $\ldots \ldots \ldots \ldots \ldots \ldots \ldots \ldots \ldots \ldots \ldots \ldots$

II ELINS ERWECKUNG $\ldots \ldots \ldots \ldots \ldots \ldots \ldots \ldots \ldots \ldots \ldots \ldots$

III DAS SONNENSPEKTRUM ................. 14

IV TOD UND TEUEEL $\ldots \ldots \ldots \ldots \ldots \ldots \ldots \ldots \ldots \ldots \ldots \ldots \ldots \ldots \ldots$

V SCHLOSS WETTERSTEIN ............... 43

VI SCHLUSS $\ldots \ldots \ldots \ldots \ldots \ldots \ldots \ldots \ldots \ldots \ldots \ldots \ldots \ldots \ldots$

BIBLIOGRAPHIE $\ldots \ldots \ldots \ldots \ldots \ldots \ldots \ldots \ldots \ldots \ldots \ldots \ldots \ldots \ldots \ldots \ldots$ 
KAPITEL I

\section{EINLEITUNG}

Freudig schwlr ich es mit jedem Schrure

Vor der Allmacht, die mich zllchtigen kann:

Wie viel lieber whr ich eine Hure

Als an Ruhm und Gluck der reichste Mann!

Welt, in mir ging dir ein Weib verloren,

Abgeklurt und jeder Hemmung bar.

Wer war fthr den Llebesmarkt geboren,

So wie ich dafllr geboren warll

So schrieb Frank Wedekind Uber sich und seine Idee der Prostituierten. Er nannte das Gedicht "Konfession". Hier kann man seine Bejahung zur Prostituierten spliren. Mit diesem Lobgesang auf die Hure verklindet er die Bedeutung des Freidenmldchens in seinem Leben, Es lst sehr eng mit seinem Leben und mit seinen Dramen verknupft. Der Weg zu Wedekinds Idee der Prostitulerten beginnt mit dem "Welb". Joachim Friedenthal glaubt, dass Wedekinds Vorstellung vom Weib seinen Anfang in seinem Helmatsort Lenzburg hat. Dort 8011 er eine Jugend, ungeben von der Natur, genossen haben. Das Naturgefthl, das sich aus dieser Jugendumgebung entwickelte, soll sich "auf besondere Art" zu einem anderen Naturgefthl gewandelt haben: die nat Urliche ursprlingliche Sinnessch bnhelt des Welbes. Dieses natUrliche Geflh1 gegentber dem Weib steigerte Wedekind soweit, dass Ihm kein Raum mehr blieb fllr die Ubrige Natur.

Von Schloss Lenzburg in der Schweiz ging Wedekind 1883 nach Mllnchen, um Jura zu studieren. Er hbrte nicht nur Vor- 
lesungen der Rechtsfakult tht sondern auch bel dem Gynlkologen Winkel. Wedekinds Interesse fllr die Sexualit dieser Zeit wlssenschaftliche ZUge zu besitzen. Dieses kHrper11che Interesse flir das Welb hat Folgen In Bezug auf seine Idee der Liebe. Das Resultat dieses Denkens spricht Paul Fechter in dem folgenden Kommentar aus: "Wedekind kennt nur elne Liebe: die zeugende, empfangende, kBrperlich bedingte, vom KHrper empfundene, auf den KHrper gehende." Wedekind plddiert selbst fllr den "Gelst des Flelsches"." Im Jahre 1910 In seiner Einleitung "AufkllHrung" zu dem Drama Totentanz tellt ex die Menschen in zwel grosse Rlassen ein: die mit dem Wahlspruch "Fleisch blelbt Flelsch--im Gegensatz zum Gelst" und die mit dem Wahlspruch "Das Fleisch hat seinen elgenen Geist". Er bekennt sich zu dem zweiten Wahlspruch. Dlesen Geist des Fleisches nennen wir im allgemeinen Erotik. Wilhelm Bolze erlHutert diese Idee der Liebe-Erotik noch welter :

Das Innere Erlebnis, das seine [Wedekinds] Seele vbilig ausfulite und ihn naturnotwending zu dichterischer Gestaltung trieb, war die bis zu ihrer Hussersten Konsequenz gestelgerte Liebe, die hellige Gottgewolltheit der Sinnilchkelt, das Mysterfum der reinen, zur h४chsten und edelsten erotischen Gemeinschaft hinaufwelsenden Hingabe. Und da Wedekind.... lelcht erkannte, dass diese seelischen Vorgunge vorzugswelse der Frau eigen sind, so wurde sein Spezialgeblet die Psychologie der ausschliessilch liebenden Frau, die er bis zu ihrer vbiligen Entartung in der Dirne verfolgte. 8

Stefan Zweig bemerkt dazu:

Wedekinds ganze Liebe geht zu jenen letzten Aufrichtigen, zu den letzten elniinigen, eindeutigen Gestalten unserer Zeit, die von der burgerlichen Gesellschaft an den Rand ihrer Verachtung gedrHngt 
werden, die sie ausstossen mochten, wenn sie ihrer doch nicht bedurften: den Hochstapler, die Hure, den Clown, die letzten pittoresken Figuren unserer ernthchterten Welt. 9

Eo gibt ooviele Namen fur eine dieser "letzten Aufrichtigen" In der Gesellschaft: Hure, Halbweltdame, demi-monde, Prostituierte, Lebedame, Kokotte, Dirne, uew. Wedekind nannte 10

oie "Freudenmadchen". Fur thn war der schlechte Ruf des Preudenmadchens in der burgerlichen Gesellochaft ein Zelchen der Heuchelel in die日er Gesellschaft. Des Freudenmadchen Ist die Verkorperung des Sinnengenusses, das die Gesellachaft "zu verstecken und zu verotummeln" versucht. Ein naturlicheo Leben kann nur von einem Menschen gefuhrt werden, der auseerhalb der Gesellschaft und ohne thre Gesetze lebt. Die Hure lebt auseerhalb dieser Heuchele1; sie lebt aufrichtig, weil aie naturlich lebt. ${ }^{12}$ Tedekind otellte Frauen aus dem Reich der Burgerlichkeit und Freudenmadchen einander gegenther und bezeichnete die Freudenmadchen als die feinfthligeren 13

Geschopfe. Aus den Notizen zu dem Entwurf seines Schauspiels Die Jungfrau ist folgendes im Zusammenhang mit der ProstitutIon und der Gesellschaft beachtenewert:

Dasg es eine hohere Gerechtigkeit alo die der burgerlichen Geoellachaft gibt, hat schon Christus gesagt und die Anerkennung seiner Behauptung der Menschheit durch seinen Tod erkauft. Vor dieser hbheren Gerechtigkeit findet das Mysterium der Prostitution ebeneo seine Erlauterung, wie das Mysterium der the oeine Erlauterung vor der Moral der btirgerlichen Gesellochaft findet.14

Wedekind sieht die Prostitution als ein "Myoterium" und alo einen Gegensatz zu der heuchlerischen Liebe und Ehe. 15 Es wurde ihm von Franz Blel vorgeworfen, dass er als ein Menech 
mit lebhaftem Interesse fur die moralischen Angelegenhelten Grinde sucht flir die ausserordentliche Sympathle, die er fur Freudenmädchen empfindet. Selne Sympathle gibt thnen Bedeutung und eine Weltanschauung, die christlich aber unsentimental geflirbt 1st. Ob Blels Vorwurf berechtigt lst, werden wir Im Laufe der kommenden Untersuchung feststellen k8̈nnen.

Wedekind liebte die Idee des Freudenmildchens, er lebte und verkehrte mit thnen oft in Paris, eriverstand sie. Inwiefern er sle verstand, werden wir in der folgenden Analyse der vier Wedekinddramen sehen. Die Untersuchung wird sich auf die chronologische, phIlosophlsche Entwlcklung von Frank Wedekinds Idee der Prostituierten beschrlnken. DIe Wahl der vier Dramen aus den vier Schaffensperioden von Wedekind 1st chronologisch, d.h. eln Drama aus jeder Perlode murde auggewthlt, um einen repräsentativen Uberblick zu sichern. 
RAPITEL II

\section{ELINS ERWECKUNG}

Im Jahre 1887 schrieb Wedekind die Verskombdie Eling Erweckung. Das Thema des Dramas lst die Unschuld. Der Held, E1In, lst Theologe und leidet an den Folgen seiner unterdrilckten, aber sonst normalen, Geschlechtsgefthle. Am Ende des Stllckes wird er von einer Prostituierten namens Ella von seiner Unterdrlickung befreit. Die Schlussszenen und die kleine Prostituierte Ella werden der Gegenstand der Untersuchung sein.

Mitten in einem philosophischen Gesprllch zwischen den Freunden Oskar und Elin, das in das unbeweisbare Metaphysische Uberzugehen droht, tritt Ella auf. Die Schilderung des tbergangs von der metaphysischen Unwirklichkelt zu der unmittelbaren Wirklichkeit donnert wie ein Hammerschlag:

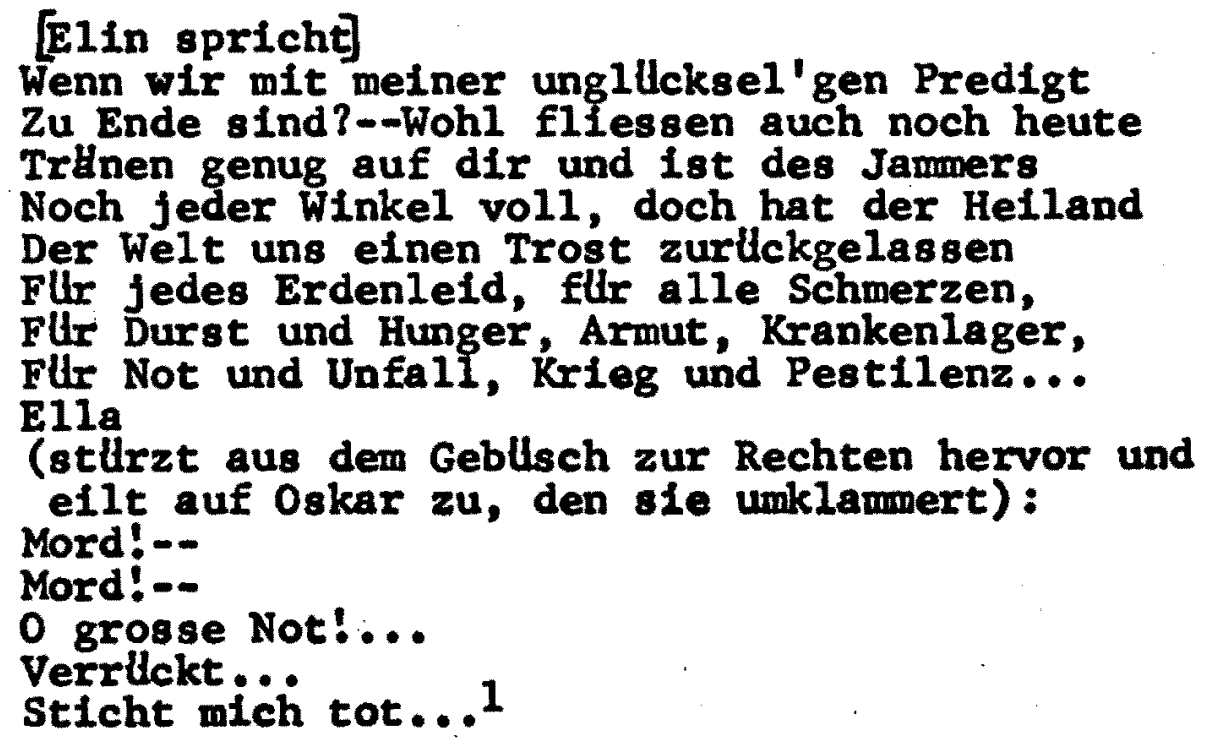


Auf elnmal stehen wir in der unmittelbaren Wirlichkelt. Die Frage ist nicht mehr ob der Heiland uns einen Trost in der Not gibt; die Frage lst Wirklichkelt geworden. Noch wissen wir nicht, wer E1la lst; sie kommt aus dem Nichts. S1e redet atemlos welter:

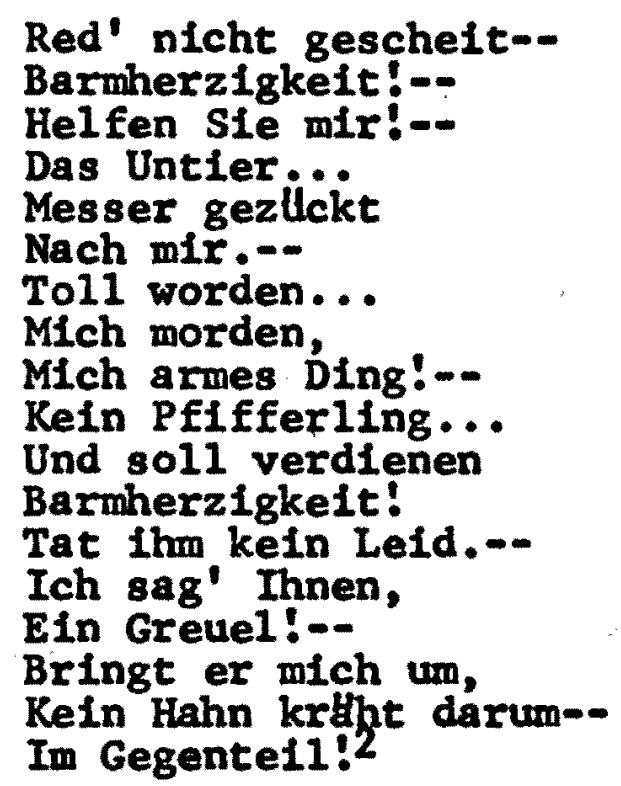

E1la hat ihre ganze Geschichte in Umrissen erzthlt. Jemand mbchte sie mit einem Messer umbringen und kein Mensch wlirde sich un ihren Tod klmmern.

Im nitchsten Moment erscheint Ellas Verfolger: Graf Schwelnitz. Als er sleht, dass Ella unter 0skars Schutz steht, will er sich zurllckziehen. Dies wird thm unmbglich durch Elins Zuruf: "Sle Lausbub Sie!" ${ }^{3}$ Ex flthlt sich als Edelman beleldigt und verlangt Satisfaktion von Elin. Aber anstatt Satisfaktion zu bekommen, wird der Graf in einen Dlalog mit Ella lber Moral und Sittlichkeit verwickelt. Ella und Graf Schweinitz sind bittere Gegner. Warum sind sie so bitter; wer sind sie eigentlich? Es wird im Laufe des Dialogs klar, dass Ella die Unschuld der natirlichen Moral vertritt gegen- 
Uber der gesellschaftlichen, die Graf Schweinitz reprlisentiert. Der negative Klang und die Etymologie des Namens "Schweinitz" IHsst schon vermuten, auf wessen Selte Wedekind steht.

Ella sucht Schutz bei Oskar und Elin und zur gleichen Zeit beschimpft sie Schweinitz' Moral: "Wenn so was nicht Moral noch Anstand kennt,/Macht's MHdchen tot, als wHren's Regenwlrmer." 5 Graf Schweinitz in seiner KapazitHt al.

Vertreter der gesellschaftlichen Moral exwidert:

Kostbar, haha!--Zum Totschiessen:--

Dies feile Fleisch weiss etwas von Moral!

Possierlich, wie in dem Abzugskanal
Noch insgeheim solch helle Tropfen fliessen!

E11a 1Hsst diese Bemerkung nicht unerwidert vorbelgehen, sie sagt:

Sle glauben wohl, well ich mit nacktem Leib Mein Brot verdien', Sie durften mich drum gleich Wie Ihren Hund traktieren?--Ich bin so wenig

Thr Hab und Gut wie Sie ein--Tugendspiegel.

Ich bin kein Rinnsal, werter Herr! Ich bin

Ein ehrlich Strassenmddchen. Fragen Sie

Die Nachbarschaft. Ich tu', was unsre Pflicht, Wle's jede tut. Auch hab' ich stets gewusst, Was Anstand helsst bel wohlerzogenen sbhnen. Wollt' mix doch eher am Waschtrog Brot verdienen, Eh' dass Ich was Untugendilches IItt', Das wider alle Sitte und Moral!

Jetzt wissen Sie's. Ich will mein rein Gewissen, Wenn ich nichts andres hab', als Schimpf und Prlgel. Und dass ich's sag'--Sie sind ein schlechter Mensch; Mbcht nicht statt Ihrer sein; Sie haben keine Moralitut.

Was meint Ella mit "Untugendliches", "Sitte und Moral" und "Sie haben keine MoralitHt"? Als Vertreterin der Unschuld, der natUrlichen Moral weist sie in dem folgenden Satz darauf hin, dass sie nur thre Pflicht tut in threr Gesellschaft. "Ich bin ein ehrlich Strassenmddchen", sagt sie. Sie tut 
nur thre berufliche Pflicht wie jede andere. Sie kbnnte sich als hellige Jungfrau geben--wenn sle wollte! obwohl niemand auf Erden thr glauben wilrde. Sie tut aber was thr naturgemiss passt. Obwohl es ihr verhHltnismbssig schlecht geht, bejaht sie reflexions 10 s des Leben. Sie hat niemale thre natUrliche Unschuld verletzt, meint sie, wenn sie von "Sitte" und "Moral" und sogar "Tugend" spricht. Graf Schwelnitz wollte Mord an Ihr verlben; das lst "unsittlich" und "unmoralisch". Ella sagt zur Erlluterung dieses Punktes:

Ich hab' geflucht. Ich hab' mich hundertfHltig

Gellsten lassen. Doch die Sittsamkelt--

So wahr Ich nur ein einfach Hurenmidchen-Ich hab' sie nie verletzt. I0

Sogar mit dem Herzog Casimir, sagt sie, wirde sie sich nicht abgeben, wenn er mit seinem Ehering bezahlen wollte. Das whre thr unsittlich. Sitte und Moral sind also die gesell8chaftlichen Eigenschaften, die Ella thr "rein Gewissen" lassen, und sie haben nichts mit threr sexuellen kbrperlichen THtigkeit zu tun.

Das Gewissen 1st eine nat Urliche Erscheinung, die Ella besitzt. Schweinitz besitzt sie nicht, denn er will morden und stehlen; er hat keine Moralittl. Das Stehlen, das Schwelnitz auslbt, ist nicht, was man unter normalem allthglichem Stehlen versteht. Ella beschreibt die Tat:

Will noch gar unmoralisch tun, zieht dann statt Sein Portmonnale das Messer, das sein MHdchen Die Zthne zeigt--das alles, well er weiss, Das MHdchen findet in der ganzen Welt Nicht einen Rechtsgelehrten, der nicht zu Gelehrt wHr', einzusehen, das $\$$ Nichtbezahlen Haargleich wie Stehlen sei...11

"Nichtbezahlen" und "Stehlen" sind flr Ella die gleichen Be- 
griffe, sle haben dieselbe Bedeutung. Flr Schweinitz,

Vertreter der blirgerlichen Gesellschaft, kbnnen sie entstellt werden, bis sle zwel verschiedene Bedeutungen zu haben scheinen: Dies ist unmoralisch und unsittlich. Seine Antwort zu der Anklage 1st nur verzweifelte Unhbflichkelt: "Du freches Mausloch!/Du Surrogat! Ich will dir, angefressnes/Reibeisen du! Du Abhub! Halbverfaultes/Kerbholz! Du Nachtquartier!" 12 Selne Flllche sind genau das, nur Fillche keine Antwort auf Ellas Beschuldigung.

Elin nimmt sich Schweinitz' Beleidigung von Ella zu Herzen. Er gibt Schwelnitz eine Ohrfeige und Schweinitz verlangt wieder Genugtuung, aber Elin verlangt erst, dass Schweinitz seine Schulden an Ella bezahle. Erstaunlicherweise will E1la den Taler nicht haben. Sle sagt: "Meln werter Herr. Verwenden Sie den Taler/Auf Thre KInder, Herr! Ich schenk' Ihn Ihren/Unschuld'gen KIndern..." ${ }^{13}$ schweinitz Hussert alch aufgeregt zu dieser von seiner Seite aus als beleidigend wirkende Ausserung:

Grosser Gott, nun hbre

Dies Ungeheuer, dieses Tier!--Nichts, nichts

Ist der verruchten Bestle hellig. Gerade

Das Helligste, das in des Mannes Brust

Sich unantastbar birgt, sein hehrstes Fllhlen, Von jeder Menschensatzung respektiert,

Das Gottverwandte, den Familienvater

Erdreistet $81 \mathrm{ch}$ dies--beinah mbcht' ich sagen,

Dies--oh, Ich finde keine Worte!--dies--

Dies--Krokodil--mit seinem schwarzen Schlamm zu

Besudeln!--Diese Lastersch1Huche!--Von

Empfindung fllr die Ideale Seite

Des Lebens nicht die Spur!--Die Polizel

Sollt' jedes dieser Schweine w bchentlich

Dreimal karbatschen lassen auf den nackten

Verflhrerleib, auf dass in dieser Weise

Sie mind'stens Achtung hegen lernen vor 
Der sittlichen Weltordnung. 14

Auf einmal redet Schweinitz wle die Seele der bligerlichen Sittlichkeit. Er nennt Ella ein Tier, ein Krokodil, einen Lasterschlauch, aber sie wollte nichts als ihr ehrlich verdientes Geld von thm haben; sle hat thm nur die Wahrheit Uber die Vernach1Hssigung seiner Fam1lie gesagt. "Die Wahrhelt" veranlasst Schweinitz' Emplrung; seine heuchler1sche burgerliche Idee der Gesellschaft gilt nur in einem Idealen Sinn, die die Wirklichkeit verneint. Die Formel Vernunft - nackte Wahrheit dient hier als ErlHuterung, denn Vernunft 1st nur ein anderer Begriff fllr das Handeln in der unmittelbaren Wirk1ichkeit. Wahrheit ist also Wirk11chkeit.

Es stimmt, wenn Schweinitz E1la ihren Mangel an Empfindung fllr die ideale Selte des Lebens vorwirft, denn E1la handelt in der Wirklichkeit. Fllr sie gibt es keine unwirkliche unvernUnftige Vorstellung der Weltordnung. Schweinitz' Heuchelei, die Unwahrhelt ist, schliesst elne vernlinftige Vorstellung der Dirne und threr Rolle In der Gesellschaft aus. Seiner sittlichen Weltordnung soll durch das Auspeltschen von Dirnen gedient werden. Dabel 1st er der einzige, der wirkliche beweisbare Verbrechen begehen wollte. Was eine Ironle, wenn er die Polizel, als Hiter der sittlichen Weltordnung, auf seiner Selte sieht!

Elin kann nicht lHnger friedlich zusehen, er verlangt nochmal den Taler von Schweinitz, aber 1Hsst thm keine Zeit zu bezahlen. Er wirft thn zu Boden und bearbeitet ihn mit 
beiden FHusten. E1In brummt: "Ker1, Ich w111 dir/DIe brave Unschuld schmthlich Ubertblpeln!" 16 Schweinitz wird jetzt selber von Elin ein Krokodil und ein Tier genannt, da Elin die Heuchelei in Schweinitz' Vorwlrfen gegen Ella einsieht. Der Trager der Unschuld und der Sittlichkeit ist E1la--nicht Schreinitz.

Warum 1st Ella die Unschuldige und die Sittliche? Dle folgende Erk1Hrung des Begriffes Sittlichkeit von Wedekind In seinem Essay "Kunst und Sittlichkeit" (1906) wird den Punkt erlHutern:

$$
\begin{aligned}
& \text { Sittlichkeit ist etwas sehr Hohes, Sagen wir } \\
& \text { einmal vorerst, dass Sittlichkeit das HUchste } \\
& \text { lst, was der Mensch erstreben kann. } \\
& \text { Sittlichkelt besteht darin, dass der Mensch } \\
& \text { das Beste, } 5 \text { ut, was er nach bestem Gevissen } \\
& \text { tun kann. }
\end{aligned}
$$

Dies hat Ella getan, deswegen ist sie die Trbgerin der Sitt11chkeit. Schweinitz denkt auf zwel verschiedenen Ebenen: die unmittelbar wirkliche und die 1deale. Nur die Heuchelei kann die Folge davon sein und nicht ein reines Gewissen. Well Ella ein Gewissen hat, ein "rein Gewissen", und nur danach handelt, handelt sie sittlich. Die Schuld kbnnte aus Ihrer Handlung nur folgen, wenn sie etwas Unsittliches unternommen hHtte. Dies hat sie nicht getan. Ella ist unschuldig, wle Schweinitz' Kinder. Sie und Ella haben das unsittliche unwirkliche Handeln nicht gelernt. Sie sind frei von der L Ulge der heuchlerischen burgerlichen Gesellschaft und deshalb schuldlos.

Elin bringt Ella, die "arme MHrtyrerin der Zivilisa- 
tIon", In Sicherheit, whicend Oskar Schweinitz bHndigt. Die vierte und letzte Szene des Dramas findet In Elins Studierstube statt. Sie beginnt mit elnem langen Monolog, in dem Elin das Wunder seiner neuen sexuellen Entdeckung himmelhoch lobt--whthrend Ella schlHft. Mitten in seinem prHchtigen, schwHrmerischen Monolog fHngt Ella im Schlaf zu reden an: "...Wer hat die magre Supp' gebraut?/Sie schmeckt nach Tausendglldenkraut.--/Brot--Sch seinem neuentdeckten Leben und Ella beklagt sich im Traum Uber die Armut; sle bittet um Brot. Das Praktische behllt sie sogar im Traum im Auge. Von einer schwltmerischen, idealen Vorstellung der Liebe glbt es bel thr kelne Spur. Ihr natlir11cher Sinn ist auf das Gegenstlindliche gerichtet. Infolgedessen 1st sie nicht hingerissen von Elins Liebesbeteuerungen, sondern antwortet mit der folgenden Ironischen Wendung:

Dles Aug'!--Der Schmach--Die milchtigen Gebłrden!-'s macht einem ordentlich das Herze schwer.-Komm, sllsser Schatz!--Wenn Ich dein Yater wir', Du mblstest $\mathrm{mir}$ eln Pfarrer werden. 21

Ellas Llebe zu Elin ist eln Weg, der sich durch ihre Rettung von selbst ergeben hat, sie beabsichtigt nicht, ihm einen besonderen Gefallen dadurch zu erwelsen, dass sie bel thm bleibt. Ella lst schon elnes der schbnen wildep Raubtiere, die Wedekind so sehr gellebt hat. 22

In dem letzten Dialog des Dramas tadelt Ella Elin als seine Rede sich im Unwirklichen zu verlieren droht:

Will mir scheinen, Bist wieder am Weinen, Gellebter Schatz.

Komm, sel zufrieden.

Haben $j a$ beide

Zu jeder Freude Reichlichen Platz. 23 
Elin soll seine Befriedigung in einer Anerkennung des realen Lebens suchen. Sein unrealistisches Schwllrmen schafft kelne Verbindung zum Alltag. Hier endet das Drama.

Elins Exweckung 1st ein Jugendwerk Wedekfinds. Es lefdef oft an schnbrkelhaftem Dlalog, der den Fluss unterbricht. Es ist noch kein reifes dramatisches Werk. ${ }^{24}$ Das Wichtigste an dem Drama 1st seine Rolle in der Entwicklung von Wedekinds dramatischem Schaffen. In Elins Erweckung "...finden sich nicht nur Gedanken, die splter tiefer und relfer weltergesponnen werden; sondern es treten auch dramatische Personen, die fllr Wedekind allmblhlich Typen werden, zum erstenmal deutlich hervor; unter ihnen ist Ella...die wichtigste." 25 In der Analyse der anderen Wedekinddramen werden wir Ella in verschledenartigen Gestalten wiedererkennen kbbaen. 
KAPITEL III

\section{DAS SONNENSPEKTRUM}

Das dramatische Fragment Das Sonnenspektrum, mit dem Untertitel Ein Idyll aus dem modernen Leben, wurde im Jahre 1894 geschrieben. "Wedekind sagte selber, dass er sein Sonnenspektrum geschrieben habe unter dem Eindruck von KHnig Sudrakas Irdenem Waggelchen, dem Indischen Schauspiel um Vasantasena..." Die belden Dramen haben wenig gemeinsam, aber die "...Begelsterung fltr das Allgemein-Menschliche und NatUrliche des Gegenstandes..." ist in beiden zu sehen. Obwohl Sudrakas Zehnakter elne Bajadere, Vasantasena, als Hauptfigur hat, betont Wedekind das Erotische stHrker. ${ }^{2}$ Wie wir in dem Vergleich der folgenden Gartenbeschrelbungen feststellen kbnnen, 1st das wesentlichste gemeinsame Element der belden Dramen die Atmosphere. Maitreya wird mit einem Auftrag zur Bajadere geschickt. Er hat das mit Juwelen, Gold und Blumen geschmilckte Haus beschrieben, dann beschreibt er den Garten :

Von hier aus ging's durch einen herrlichen Garten: fremdartige Büume in vollem Billtenflor. Zwischen thren StHmmen seidene Schaukeln, auf denen sich kindliche Bajaderen wiegten. Rote und blaue Lotosblumen splegelten sich In dem stilien Welher dleses Freudenhains. 3

Und jetzt WedekInds Gartenbeschrefbung:

Eine klthle, schattige Allee aus breiten Platanen. Der Boden ist mit feinem gelbem Sande bestreut. 
Zwischen den hellen Baumsthmmen stehen dunkelgrline BḦnke. Rechts vorn elne kleine Rotunde mit einer halbkreis flrmigen Marmorbank. Hinter der Allee im grellsten Sonnenlicht ein runder Rasenplatz mit einer Schaukel und einer Wippelbank. 4

Nun tritt die "kindliche" Bajadere, Franziska, auf,

Franziska und Gregor treten von links in die Allee.--Franziska trlygt ein dunkelgrtines seidenes Hemd mit weissen Schleifen, hellgrlne Strlimpfe, welsse Ballschuhe und eine weisse Schleife im offenen Haar.5

Die Atmosphtre in beiden Beschreibungen lst bunt, kindlich und durchaus Idy1118chen Charakters. Man erwartet nur Freude in solch elner Umgebung.

Die Bewohner dieser Insel der Freude sind kleine Prostituierte im Durchschnittsalter von etwa 18 Jahren. Sie haben eine "Madame", die thnen gleich Mutter und Vater 1st, und sie helssen: Melitta, Franziska, Rebekka, Schneewittchen, Minehaha, Scharolta, Radudja und, optter In dem Drama, Elise. Ihr Hrztlicher Betreuer lst ein Dr. Puslowsky. Man merkt, dass es acht Mtdchen sind, wie die acht Farben des Spektrums. Zur Erlduterung der Spektrummetapher wird die folgende Erk1trung von Friedenthal beitragen:

...erkannte man nicht erst durch die Farben der Spektral-Analyse Leben und Wesen der Sonne? So erkenne man durch die vieldeutigen Farben dieser spieler18chen Spektral-Analyse Sonnenspektrum ein Stllck vom Leben und Wesen der ewigen Sonne, die dahinter steht: der Sternen-Urgewalt Llebe. 6

Die Liebe, die wir in der Elnleitung gesehen haben, lit fur Wedekind k8rperlich bedingt. Da das "Leben und Wesen" dieses Idy11s dle Llebe 1st, 1st es nicht erstaunlich, dass das Idyll 
sich mit einer Verherrlichung der Liebe befasst, wie sie in allen bunten Farben des Spektrums existiert. Das Stllck 1st eine "Verherrlichung des Lustprinzips", das die Prostitulerte verkbrpert. Ein ntheres Betrachten des Dramas wird dies bestlitigen.

Die erste Szene des Idy118 ist ein Dialog von witzigem Inhalt, in welchem Wedekind einen schon erw thnten Gedanken Hussert :

GREGOR Wird denn die Liebe nicht schliesslich auch langwellig?

Franziska schlttelt den Kopf.

GREGOR Das darfst du natlirilch nicht sagen. FRANZISKA Ist sie Ihnen langweilig?

GREGOR Ich bin leider kein hlbsches MHdchen. FRANZISKA Je mehr man liebt, um so mehr mbchte man lieben.

GREGOR Wenn ich das auch von mir sagen kbnnte!

FRANZISKA Sie sind nicht freundilch.

GREGOR Ich beneide dich. WHre Ich als hllbsches

MHdchen zur Welt gekommen, ich hHtte ${ }_{8}$ voraussichtlich auch deinen Beruf erwithlt.8

Wie in allen anderen Dramen scheint auch hier die verkleidete Gestalt des Dichters durch. Wedekind bedauert, dass er nicht als htbsches MHdchen zur Welt gekommen ist. Seine Vorstellung der prostituierten Liebe lautet freudig und zuglelch sachlich: "Je mehr man liebt, un 80 mehr mbchte man 1leben." Im nllchsten Dialog zwischen Madame und Dr. Puslowsky merken wir die Einflhing eines anderen Elements: die reale Gegenwart.

In diesem Dialog unterhalten sich Madame und Dr. Puslowsky ther eine $16 \mathrm{j}$ thrige Prostituierte, die $8 \mathrm{chwer}$ erkrankt 1st. Dr. Puslowsky versucht, die Madame zu beruhigen, aber sle hbrt nicht zu. Die AtmosphHre des ganzen Dlalogs wird 
gleich von Madames erster Kusserung bestimnt:

Die ganze Nacht hat sie phantasiert. Ich meinte zweimal: Jetzt ist es aus. Sie 8 ah mich 80 furchterlich an. Sie sagte, wepp sie doch nochmal von vorne anfangen kbnnte. 10

Wir erwarten jetzt eine Art blirgerliche Sentimentalit

vielleicht eine Bekehrung der Madame. Etwas ganz anderes folgt jedoch :

DR. PUSLOWSKY Das ist ein bedenkliches Symptom. MADAME Das habe ich mir auch gesagt. Sie nahm mich fllr thre Mutter. Sle hatte die Hunde liber dem Kopf; sie war wie sechs juhrig. Nein, dieses MHdchen! Ich hltte thr eine Puppe holen mbgen. DR. PUSLOWSKY Hat sie denn ordentlich Offnung gehabt?

MADAME Danke. Vorgestern war's noch ganz respektabel. Aber sie hat ja keinen Appetit. Ich habe ihr Bouilion mit Kibschen hinaufgebracht. Sie erkennt es nicht. 11

Wie wir merken, zweifelt Madame keinen Moment an ihrem Berufe. Sie diskutiert nicht den Satz, "...wenn sie doch nochmal von vorne anfangen kbnnte". Madames Interesse an der kranken Prostituierten ist mlltterlich. Ihr Interesse an threm gemelnsamen Beruf selbst ist sachlich, daher die kuriose Mischung von MUtterlichkelt und Sachlichkeit in dem Dialog, der die beiden Begriffe behandelt. Zwischen jedem Ausbruch der Sent1mentalitHt werden wir mit der grbssten Sachlichkeit in die Wirklichkelt, die reale Welt, zurllckgebracht.

Dr. Puslowsky redet welter von anderen derartigen FHllen, die er bel Madame behandelt habe. Sie will aber nichts davon hbren, denn sle denkt immer aufgeregter an die kleine Prostituierte. Der Dialog wird fortgesetzt:

MADAME Die anderen? Schweigen Sie mir. Das

kennt ja nichts als fressen und schlafen. 
Da relsst man mittags zum Essen die Glockenzllge in Fetzen, und nachts um viere finden sie vor Betrunkenheit das Bett nicht. Da liegen sie dann und wissen nicht, wie sie getauft sind. Das ist eine Hurenkompanie, die mir der Allmblchtige zu meiner irdischen Ltuterung beigegeben. Den Champagner saufen ole elnem unter der Nade weg und mit meinem gediegensten Klienten machen aie Verschwbrung. Sie? 0 Gott, - Gott, wenn ich denke, wie sie jetzt da oben im Fieber liegt... DR PUSLOWSKY Sie hat ja nat lrilch auch des Guten ein wenig zu viel getan? MADAME Was wollen Sle damit sagen, iHerr Doktor? Ich zwinge niemanden. Sie hat sich nicht nbtigen lassen. Sie war Immer die erste, und die letzte. Das ist nun thr Dank dafllr. Es lst entsetzlich. Je besser der Mensch sich macht, um so schlechter geht es ihm. Da habe ich alte kalfaterte, kalmutterte Ungeheuer, die auf der Welt nichts kennen als ihren Geburtsort, und werden mit jedem Tag fetter, und 80 ein armes unschuldiges Menschenkind muss yon heute auf morgen elend zugrunde gehen! 12

Madame teilt Ihre Geflhle der Mltterlichkeit wit denen dex Ungerechtigkeit. Die Kleine war fllr Ihren Beruf vortrefflich geeignet und tat Immer ihre Pflicht. Die anderen Uben ihren Beruf nur aus, um zu geniessen. Die Ungerechtigkeit liegt darin, dass die Unschuldige sterben muss und die anderen, die ohne berufliche Pflicht leben, das Leben welterhin geniessen durfen. Zum zweiten Mal sehen wir die Rolle der Unschuldigen von einer pflichtbewussten Prostituierten gespielt. Sie ist ein "armes unschuldiges Menschenkind", weil sie sttndig ihre Pflicht getan hat. Well sie sich zu oft hingegeben hat, ist ihr Nervensystem vollkommen zerrlittet. Dieser Punkt ist wichtig, denn sie ist das Opfer einer nur physischen Krankheit, der Nymphomanie, und leidet nicht unter 
einer seelischen Zerrlittung. Der Beruf der Prostituierten verursacht physische, nicht seel1sche Krankheit. Mitten in elnem Idy11 erleben wir eine negative Selte der Prostitution. Die reale Gegemwart macht sich bemerkbar.

Das Drama setzt sich im spielerischen Ton fort. Heinrich und Melitta spielen auf einer Wippelbank. Scharolta und Rebekka, Max und Theophil unterhalten sich in dem Idyllischen Garten. Folgendes ist elne Beschreibung der Kleider, die die vier jungen Menschen tragen. Wedekind legt grossen Wert auf Atmosphlire in diesem Drama. Deswegen ist die Kleidung so wichtig fur thn.

Max und Theophil, von Rebekka und Scharolta gefolgt, treten von links in die Allee. Max in Zylinder, schwarzen Gehrock, hell karierten Beinkleidern, welsser Weste, Lackstiefeln, hohem Stehkragen und weisser Krawatte. Theophil in kamelbraunem JHgerkostim, trigt goldene Brille und lockIgen rbtilichblonden Vollbart. Rebekka mit grliner Schleife im Haar, hellgrinen Strlimpfen, dunkelgr Unen Schuhen und Strumpfbłndern; Scharolta mit roter Schleife im Haar, gelben Strlimpfep, roten Schuhen und grllnen
Strumpfbundern.

Diese Beschreibung gleicht einer naturalistischen Beschreibung. Wedekind wollte einen gewissen Effekt mit dieser Genauigkeit erzielen: die Sinnlichkeit. Diesem Zweck dienen die grellen Farben, die Hemden, die Schleifen und die Strumpfblinder. Eine reizendere Welt hlltte sich niemand ausdenken kbnnen!

Das Paradies wire nicht vollkommen ohne einen slld1Hndischen ObsthHndler--er heisst in diesem Fall Perugino und verkauft Trauben, Oliven, Datteln, Feigen usw. Er schreit: 
"Orangen, meine Herrn, meine schUnen Damen! Kandierte

Datteln! Frische Feigen!", Er singt: "Er nahm mich um den Leib und lachte/Und fillsterte, es tut nicht weh...." 14

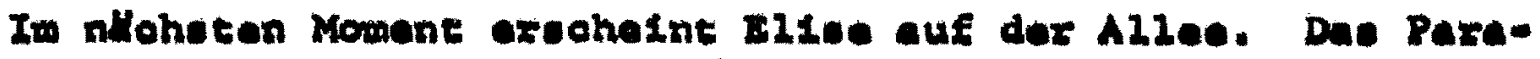
dies bekomnt einen neuen Pfau.

Elise 1st offensichtlich aufgeregt und spricht hastig. Franziska und Gregor beobachten sie und Gregor flyngt wit thr 2u reden an:

GREGOR Sie zittert. Sprechen wir ein wenig mit thr. (zu Elise) Wollen Sie hier in Dienst treten?

ELISE (sieht thn mit grossen, gewitterschweren Augen an) Ich kann nicht anders. GREGOR Das kann Ich Thnen nfght verdenken. Ich kann auch nicht anders. Is

Was meint Elise mit den Worten "Ich kann nicht anders"? Warum muss sie in Madames Dienst treten? Gregor und Franziska fragen sie aus und untersuchen ihren Kbrper:

GREGOR (zu Elise) Also nur Liebhaberei?

Elise droht in die Knie zu sinken.

FRANZISKA Da haben wir es nun.

GREGOR (Elise zu einer der BHnke flhrend)

Das ist nicht so schlimm. Das zeugt von

gesunder Natur. Das Korsett braucht man

ihr nicht erst aufruhaken. Sie trHgt keins.

(Zu Elise) Sei ruhig, Midchen. Hier tut

einem niemand ein Leid. Hier weint man nur

Freudentrdnen.--Ich will thr aber doch die

Taille ein wenig aufkndpfen...

Franziska hat sich rechts neben Elise gesetzt und

betrachtet sie aufmerksam. Sie wimmert, als

mllsste sie auf acht Tage genug haben.

GREGOR Sie hat schßne Augen. (Elise die

Taille $\forall$ ffnend) Wie das dampft!--

Man sieht es aufstelgen.--Es lst thr

alles zu eng. 16

Wieder hbren wir Wedekind durch Gregor sprechen. Er gibt die Philosophie des Freudengartens kund: "Hier tut einem niemand 
ein Leid. Hier weint man nur Freudentrünen." In der Tat wird Elise nicht roh oder wollustig behandelt, sie empfungt Mitgefthl und Verstlundnis flir ihre Situation. Wedekinds Darstellung von Elises Grund in Madames Dienst zu treten, zeigt dem Zuschauer Elises sexuelle Erregtheit. Madame wird gerufen, um diesen Fall zu untersuchen. Sie tritt sehr erregt auf und hHlt in Dialogform einen Vortrag Uber thre Bezlehung als Madame zur Gesellschaft:
MADAME (die Schultern zurllckwerfend) Was haben Sie hier zu tun! ELISE Ich wollte fragen...
MADAME Ob man hier dem Herrgott den Tag abstehlen kann und mir auf der Nase herumtanzen! ELISE 0 Gott im Himmel...
MADAME Und hat man mich elend und arm gefressen, dann nimt mich die Obrigkeit bel den Ohren und reklamiert Menschenwlirde und Christengllick und der Henker weiss, was fllr Kostbarkeiten, wo die Person keinen Strumpf mit ins Haus gebracht hat!17

Madame weist darauf hin, dass sie der Mensch 1st, der sich um das Schicksal eines armen MHdchens klmmert; die Gesellschaft. tut nichts als nach Madames menschlicher Tat zu reklamieren. Die "Menschemwllrde und Christenglllck" sind "Kostbarkeiten", die sie sich nicht leisten kann, denn die beiden haben keine Beziehung zur Menschlichkeit. Sie hat es satt sich fur anderer Welber SchHferstlindchen zu schinden und ihnen ein komfortables Familienheim zu geben, damit sie sle dem Scharfrichter ans Messer liefern. Nur Gutes tut sie, und da ihre Taten sich nicht mit der Heuchelei der burgerlichen Gesellschaft vertragen, erntet sie Schlechtes.

So kommt es oft vor, dass MHdchen, die sich bel Madame 
melden, nicht einmal einen Taufschein haben. Dieses Mal wird Madame jedoch nicht enttHuscht, denn Elise hat einen Taufschein. Nach Elises Versicherung, dass sie weder eine Schwangerschaft noch sonst etwas Unerwinschtes mitbringe, bereut Madame ihre Beschimpfungen. Aber sofort danach werden die Hausregeln fir Elise kristallklar ausgelegt:

Aber das lassen Sie sich gesagt sein, wenn Sie sich volltrinken und zetermordio schreien und interessante Anfulle bekommen und thernehmen und mir hier schlechter Laune sein:wollen, dann sind wir bald miteinander fertig. Ich Ubernehme meinen Glsten gegenlber die Verantwortung, dass sie mich an Leib und Seele erfrischt verlassen und mblichst bald und oft wiederkommen. Und da kenne ich keinen Unterschied der Person. Mir gilt jeder Mensch gleich. Was dem einen recht ist, ist dem andern biliig. Wenn sie etwa darauf ausgehen und wollen sich hier verlieben und mir einen alten treuen Milliondr unter den Augen weghelraten, dann brauchen Sie sich gar nicht erst auszuzlehen. Dann machen Sie nur stracks, dass Sie mit Thren Herrlichkeiten wieder zum Tempel herauskommen.

ELISE Ich will keinem etwas verwehren.19

Von Verwehren will Madame nichts hbren. Sie entgegnet:

Wenn Sie hier verwehren wollen, dann lassen Sie sich als Stlitze der Haus frau engagieren! Wenn meine GHste thr Geld ausgeben, dann wissen sie wofllr. Die gottselige Zuversicht kbnnen sie bel Thren Kusinen zu Hause billiger haben.20

Hiermit gibt Wedekind seine Philosophie der Prostitution kund: Sie besteht aus sechs Punkten, d.h. Regeln fllr Prostituierte: Sie mllssen 1. ehrlich und missig leben, 2. sich des Berufes erfreuen, 3. die Ghste kbrperlich und seelisch erfrischen, 4. keinen Unterschied zwischen Kunden kennen, 5. Ihrem Beruf treu bletben, und 6. blirgerliche Vorstellung der Prostitution zu Hause lassen. Damit zeigt uns Madame, dass die Prostitution 
kein scherzhafter Gegenstand ist. Dafulr sind die Regeln viel zu sachlich und wohl Uberlegt. Als Seitenhieb kann der letzte Satz verstanden werden. Madame zeigt stolz auf ihren Beruf, der Ihrer Melnung nach im Gegensatz zur bltrgerlichen Ehe steht: Ihre Prostituierten 11eben nicht, um sich der "gottseligen Zuversicht" zu versichern! Sie lieben um der Liebe willen.

Elise wird engagiert und es werden ihr ein Hemd und andere Kleidungsstllcke gegeben. Ein neuer Kunde, Edgar, tritt auf. Madame sammelt Ihre Midchen zu sich und Edgar 8011 whlen. Madame Hussert sich dazu:

Mehr habe Ich Ihnen nicht zu bieten. Mein Haus ist ein Tempel der Freude und der Gesundheit. Ich wlinschte sehr, dass es dem Herrn gellinge, das Ideal seiner Trtume hier bel mir zu verwirklichen. 21

Wie Huri in islamischen Himmel latcheln Edgar die MHdchen an. Wie begierig sie sind ihren Beruf auszulben. Hier wird die Liebe geschltzt und verherrlicht. Hier wird keinem Kunden Grund zu Klagen gegeben.

Im nächsten Auftritt werden wir wieder in die Wirklichkeit zurllckgefthrt. Dr. Puslowsky erzth1t Madame, dass er nichts mehr flur die kleine sterbende Nymphomanin, Minetta, tun kann. Madame erwidert:

Die Guten sterben jung. Sie whre sonst eher heimgerufen worden. Die eine geht drauf, weil sie all ihre Jugendkra ft in sich hineinwhrgen muss; die andere geht drauf, weil sie den Anforderungen, die die heutige Gesellschaft an ein junges MHdchen stellt, nicht gewachsen 1st. 22 
Madame nennt Minettas Nymphomanie "die grenzenlose Leidenschaft" 23 , und gerade diese Leidenschaft, meint sie, ist fll Minettas Zerrlttung verantwortlich. Minetta muss starben, well thre grenzenlose Leidenschaft keine andere Ausdrucksmbglichkeit in der burgerlichen Gesellschaft findet. Den gesellschaftilchen sexuellen Anforderungen war sie nicht gewachsen. Die Prostitution war ihr einziger Ausweg. 25 Dies selbe Problem wird in der nüchsten Szene in etwas anderer Gestaltung behandelt.

Elise wird von den anderen Prostitulerten zu Dr. Puslowsky zur Untersuchung gebracht, und sie muss vor diesem Reprobsentanten der Regierung schwbren, dass sie freiwillig in das Freudenhaus eintreten will. Wie ein Donnerschlag wirkt die Nachricht, dass Elise noch Jungfrau 1st. Das Interesse an dem Grund, weshalb sle Freudenmldchen werden mbchte, wird In allen Amwesenden erweckt. Dr. Puslowsky fragt sie aus:

DR. PUSLOWSKY --Hat Sie irgendjemand gezwungen, hierher zu gehen?

ELISE Nein.

SCHNEEWITTCHEN Sie muss blelben! Wir lassen sie nicht wieder fort!

DR. PUSLOWSKY Sel ruhig! Es hat niemand thren freien Entschluss zu beeinflussen.

(Zu Elise) Sagen Sie offen, haben

Sie sich aus eigenem Antrieb in dieses Haus gefluchtet?

Elise schweigt.

DR. PUSLOWSKY Selen Sle mutig! Fassen Sle sich ein Herz! Ich frage sie nicht, ob Sie gegen Ihren Willen hier sind.

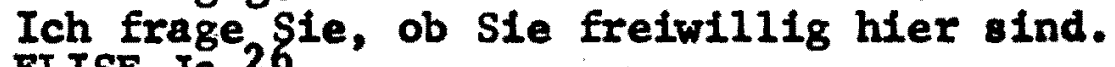
ELISE Ja.26

Elise, die frther Angst hatte, dass sie nicht in Madames Dienst treten durfte, verwelgert eine Antwort auf die Frage, 
ob sie "freiwillig" und aus elgenem Antrieb zu Madame gekommen sel. Eine unverstundliche Situation wird mit einem scheinbaren Paradox erldutert. Die Frage, ob Elise freiwlllig da sel, wird von ihr bejaht, sle ist aber in Wirklichkeit gegen ihren Willen im Freudenhaus. Die Lbsung liegt in Wedekinds Motto: Das Fleisch hat seinen eigenen Geist. Elises Fleisch hat sle dorthin zu Madame getrieben, ihr Wille (Gelst) war dagegen. Elise ist mit threm von Gott geschaffenen freien Willen dort. In der Gesellschaft fand sie, wie Minetta, keine Erlbsung threr natUrlichen Geschlechtstriebe.

Der gute Doktor setzt seine Untersuchung fort. Die Geschichte von Elises Kampf mit ihrer sexuellen Entwicklung entfaltet sich. Da es Elise nachts zu warm war, musste sie immer ihr Nachthemd ausziehen.. Sie wusste nicht, woher die WHrme kam. Es wurde thr täglich wHrmex, bis sie eines Abends aus dem Fenster stieg und auf den Apfelbaum kletterte, wo sle vor Verzweiflung die ganze Nacht blieb. Sie erzthlte, wie ihre Mutter sie fragte, ob etwas 108 whre, als sie in der Nacht im Bett schrie. Auf Elises Antwort, "ich kann nicht schlafen", sagte ihr die Mutter, sle soll bis tausend zthlen. Am ndlchsten Morgen lief sie zu Madame.

Thre Geschichte schluchzend zu Ende erzthlend, wird Elise von Madame und Dr. Puslowsky getrbstet. Die Aphorismen, die ' er so oft In seinen Dramen benutzt, kommen jetzt eins hintereinander: "Es sind viele berufen, aber wenige sind auserwhlt" ${ }^{27}$, meint Dr. Puslowsky in Zusammenhang mit Elises neuem Beruf. "Und merke dix", sagt Madame zu Elise, "es 
gibt eben nur einen Weg in dieser Welt, un wirklich glllcklich zu sein, das ist, dass man alles tut, was man kann, um andere so gltlcklich wie mbglich zu machen." 28 Und am wichtigsten von allem ist Dr. Puslowskys Ausserung ther die wahre Kunst: "Was die wahre Kunst ist, dass muss sich ganz aus sich selbst entwickeln. Alles Ubrige, und wenn es noch 80 sch bn mit den Gesetzen klappt, 18t nichts als Prostitution." 29 Diese Worte spricht der Doktor zu Elise tber den Dichter Eoban, der gleich auftreten wird. "Durch" diesen Vergleich der Kunst des Dichters mit der des Freudenmidchens wird die Aussbhnung von Kunst und Natur intendiert." 30 Die Kunst sol1 die nat Urliche Schbnheit und Sinnenfreude verherrlichen. Eobans Lieder reflektieren dieses Prinzip. Ein Beispiel genligt, um dies zu zeigen:

[a1le Mudchen singen] Ich war ein Kind von vierzehn Jahren, Ein reines, unschuldvolles Rind, Als ich zum erstenmal erfahren, Wie sliss der Ilebe Freuden sind.

Er nahm mich um den Leib und lachte Und fllisterte: Es tut nicht weh-Und dabel schob er sachte, sachte Mein Unterr bckchen in die H४h'.

Selt jenem Tag lieb ich sie alle, Des Lebens schbnster Lenz ist mein Und wenn ich keinem mehr gefalle, Dann w1ll ich gern begraben sein.31

Die beiden Begriffe "Schbnheit" und "Sinnenfreude" werden in diesem Gedicht verherrlicht. Eoban tritt auf und wird von allen MHdchen geneckt, bis er noch ein Lied singt. Es helsst "Das Lied von der Keuschheit" und sein Inhalt lst wie folgt: 
Ein armes MHdchen wandert durch die Strassen, ohne etwas zu essen. Sie bricht zusammen vor einem Herrn, der sie auf sein Zimmer bringt. Aus Dankbarkeit fll ihre Rettung von "roher Begier" in der Stadt, will sie sich ihm hingeben. Ex untersagt ihr Angebot mit den folgenden Worten:

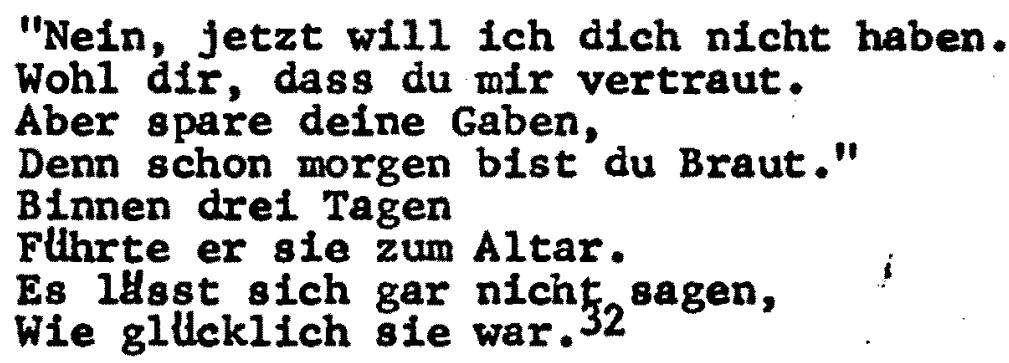

Mit diesem letzten ironischen Satz ist Elise zu Eobans Fllssen in krampfhaftes Schluchzen ausgebrochen. Damit endet das Drama fragment.

Das Sonnenspektrum wirkt wie ein Kaleidoskop auf den Zuschauer; ein buntes Durcheinander, das trotz eines schimmernden Schwankens uns doch momentane Muster bietet. Es herrschen die Freude und die Natur in dieser schillernden Welt. Niemand hungert, weder physisch noch seelisch, und Kunst und Natur haben sich versbhnt. Das Freudenmbdchen ist nicht lHnger ein Gegenstand der Verachtung oder eine Zielscheibe flir schmutzige Witze; sie ist das Zentrum, die ReprHsentantin eines hbheren Glucks: die Sinnenfreude. Durchstrbmt von utopischen Idealen beschlftigt sich das Stllck immer noch mit der Wirklichkeit. "Das Sonnenspektrum zeigt die Versbhnung der utopischen Idylle mit der realen Gegenwart." 33 Dass das Drama Fragment geblieben ist, weist auf Wedekinds Unentschlossenheit zum Thema der Prostitulerten hin. Das 
ndchste Drama Tod und Teufel zeigt die Prostituierte in einem viel dunkleren Licht, als dieses freudige Fragment sie gezelgt hat. 
KAPITEL IV

TOD UND TEUFEL

Tod und Teufel: Totentanz in drel Szenen (1905) erschien elf Jahre nach Wedekinds Sonnenspektrum. Der frUhliche gutgelaunte Ton ist jetzt hin; der Zuschauer wird sich mit einem philosophischen, triebhaft-dunklen Bild der Prostituierten zurechtfinden mllssen. Die Wandlung von Wedekinds philosophischen Ansichten 1st offensichtlich. Das Krankhafte in Beziehung zur Prostituierten, das wir im Sonnenspektrum so deutlich erkannten, erscheint in Tod und Teufel als eines der Hauptmotive. Die Verherrlichung der Sinnlichkeit spielt in diesem Drama keine Rolle.

Wedekinds Vorbild fllr die Hauptigur, Casti Piani, war Goethes Mephisto. Das allgemeine Thema des Werkes ist der Pessimist, der seinem eigenen Pessimismus zum Opfer fullt. Casti Piani, der Mddchenhdndler, hat als Gegenspielerin Frbulein Elfriede von Malchus. Sle ist Frauenrechtlerin und ist ins Freudenhaus gekommen, um thre ehemalige Dienerin, Lisiska, von der Prostitution zu retten. Lisiska hat oft nachts Frthulein Malchus' Arbeitszimmer betreten, wie das Frdulein erk14rt, "...und trdnkte seine liebesdurstige Einbildungskraft aus meinen aufgestapelten Bllchern lber die Beklimpfung des MHdchenhandels mit den verfllhrerrischsten Bildern des Sinnengenusses und der furchtbarsten Laster." Der sich daraus entwickelnde Dialog fthrt zu dem wichtigen 
Zwischenspiel, in welchem Lisiska und Herr KHnig Cast1 Planis und Frllulein Malchus' Ansichten ther die Prostitution Hndern.

Bevor das Drama analysiert werden kann, muss Wedekinds Absicht in Bezug auf den Dialog zwischen Fralulein Malchus und Cast1 Plani erwthnt werden. Er schrieb:

Die von den beiden Hauptpersonen ausgesprochenen Anschauungen tber Frauenemanzipation und kHufliche Ilebe wollte ich weder als richtig noch als massgebend hinstellen und liess die deshalb Im Verlauf der Handlung auf das felerlichste widerrufen. 3

Dieser Widerruf seiner Anschauungen ther die Frauenemanzipation und die Prostitution weist auf die exnste Natur des Dramas hin. Schon vom Titel bekomnt man den Eindruck, dass das folgende Schauspiel mehr als ein Schauspiel ist. Was uns in der Tat begegnen wird, ist elne philosophische Auseinandersetzung zwischen Casti Piani (Wedekind) und seine Anschauungen Uber die Sinnlichkeit. Wie wir Im Sonnenspektrum gesehen haben, ist auch hier die Prostituierte die TrHgerin der Sinnlichkeit. Was mit ihr passiert, entscheidet, welche Ansichten tber die Sinnlichkeit fllr Wedekind in seinen spłteren Dramen gelten werden. Denn dieses Drama ist, wie die meisten seiner Werke, eine Stufe in seiner dramatischen und philosophischen Entwicklung. Der verkleidete Dichter ist wieder einmal zu erkennen.

Nachdem Fräulein Malchus Cast1 Pian1 den Zweck ihres Besuches offenbart, d.h. Lisiska von der Prostitution zu retten, beginnt der Kampf zwischen den belden Hauptfiguren. Cast1 Pian1 hat Frtulein Malchus gerade erzthlt, dass Lisiska 
durch seine Annonce in der Tageszeitung, wie all die anderen MAdchen, zu ihm gekommen sel. Das Frylulein ist ther Casti Planis sachliche Beschrelbung seiner Methoden empbrt. Er erwidert, dass er die Ursachen ihrer "hbllischen Aufgeregtheit" wisse: Sie 1st 28 Jahre alt und noch Jungfrau, dies zeige, dass sie "...eln sehr geringes Mass von sinnlichem Empfinden..." habe. Dies schwache Empfinden befriedigt sie durch thre BetHtigung in dem Internationalen Verein zur BekHmpfung des MHdchenhandels, meint er. Da Frtulein Malchus wieder empbrt 1st, erklurt Casti Piani seine Bemerkung weiter:

Aber sehen Sie, von diesem Standpunkt aus betrachtet (vom Standpunkt des Muddchenhandels), stehen wir beide einander naher, als Sie es sich in Ihrem kleinbirgerlichen Tugendstolz jemals trHumen liessen. Ihnen hat die Natur nur eine Husserst kHrgliche Sinnlichkeit verliehen. Mich haben die Stlrme des Lebens lungst zu einer schauerlichen Einbde gemacht. Aber was fur Ihre Sinnlichkeit die Bekdmpfung des MHdchenhandels ist, das ist flur meine Sinnlichkeit, falls Sie mir etwas der Aft noch zugestehen wollen, der MAdchenhandel selbst. 7

Ihm wird vorgeworfen, dass er MHdchen verhandelt, nur um ein gutes GeschHft zu machen. Die Erwiderung:

Ein gutes Gesch dft! Selbstverstundlich! Aber gute Geschufte beruhen auf beiderseitigem Vortell! Andere Geschifte als gute mache ich therhaupt nicht. Jedes andere GeschHft ist unmoralisch! --Oder glauben Sle vielleicht, der Liebesmarkt sei für das Weib ein schlechtes GeschHft? 8

NatUrlich mbchte Frtulein Malchus wissen, was er damit meint, denn Cast1 Plani spricht Uber Dinge, die sie schon lungst geklurt hH1t. Er berUhrt den Pol, der der burgerlichen Gesellschaft entgegengesetzt steht. Sein Argument in logischen ZHgen sieht so aus: Wenn ein Mann sich in Not befindet, kann 
or atehlen oder verhungern. Eine Frau kann inre Liebe verkaufen. Sie kann dies tun, weil sie nichte bei der Gewhrung ihrer Liebesgunst empfinden muse. Der Mann ist dem Weib von Natur aus Uberlegen, weil daa Weib Kinder unter Schmerzen geblren muss. Fralulein Malchus trägt zu dem Argument etwas bei in ihrer Bestatigung der Uberlegenheit dea Mannes in Sachen der Geburt. Sie findet es bejammernowert, dass Geburtsachmerzen auch der armen Frau aufgebüdet sind. Cast1 Piani versichert ihr, dass er der gleichen Meinung sei. Er wirft ihr thre THtigkeit in dem Verein zur Bekampfung des Madchenhandels vor, in welchem sie diesen geringen Vorzug des Weibee bektmpft. Wenn der Verein siegen whrde, hatte ein armes Weib keinen Ausweg in der Not. 9 Des Madchenhandlers Wut entwickelt sioh voll. Der soziale Sinn des Dialogs wird deutlioh auggedrlokt:

Schleudern Sie Ihre Vorwllefe, wenn Sie die Bedrtlckung Ihres unglucklichen Geschlechts bektmpfen wollen, der burgerlichen Gesellschaft ino Gesicht! Bekampfen Sie, wenn Sie die Naturrechte Ihrer Schwestern verteidigen wollen, zuerst den Internationalen Verein zur Bekampfung des Madchenhandels! 10

Zum dritten Mal ist Fraulein Malchus emport. Dieses

Mal versucht oie das Freudenhaus zu verlassen. Kaum ist sie aufgestanden, packt Cast1 Plani ale bel der Gurgel und echleudert sie in einen Polsterśessel. Er lot nicht fertig mit seinem sozialen Vortrag. Feurig setzt er seine Rede fort:

Sie haben in Ihrem Leben so unendilch viel Unnutzes zur sittlichen Hebung der FreudenMadchen getan! Tun Sie doch endlich einmal etwas Nutzliches zur sittlichen Hebung der 
Freude! Dann brauchen Ihnen die armen Gesch bpe nicht mehr Leid zu tun! Weil der Freudenmarkt als der gemeinste, schandbarste aller Berufe gebrandmarkt ist, geben sich die MHddchen und Frauen der guten Gesellschaft einem Manne lieber umsonst hin, als dass sie sich ihre Gunste bezahlen lassen! Dadurch entwlirdigen diese MHdchen und Frauen ihr eigenes Geschlecht in der gleichen Weise, wie ein Schneider sein Gewerbe entwlidigt, der seinep Kunden die Kleider umsonst liefert!ll

Die burgerliche Gesellschaft ist verantwortlich fllr den schlechten Ruf der Prostitution. Die anstundige bllrgerliche Frau entwlirdigt ihr Geschlecht durch die Aufopferung ihres einzigen Vorzuges: die Fthigkeit, ihre Liebe verkaufen zu kbnnen.

Allmblich wird Frtulein Malchus von Casti Piani Uberzeugt. Ihre einzige Frage lautet: Was passiert mit einem Kind, das im Freudenhaus erzeugt wird? Die Antwort lautet Uberraschend: "Sorgen Sie daflir! Oder haben Sie als Frauenrechtlerin vielleicht etwas Wichtigeres in dieser Welt zu tun?" ${ }^{12}$ Wie das uneheliche Kind mit den Frauenrechten zusammenhHngt, erkidrt er sturmisch:

Solange ein Weib unter Gottes Sonne noch fllrchten muss, Mutter zu werden, bleibt die ganze Frauenemanzipation leeres Geschwdtz! Mutterwerden ist fllr das Weib eine Naturnotwendigkeit wie Atem und Schlaf. Dieses angeborene Recht hat die burgerliche Gesellschaft dem Weibe in barbarischer Weise verklirzt. Ein uneheliches Kind ist schon beinahe ebenso grosse Schmach wie der Liebesmarkt! Dirne hin, Dirne her! Der Name Dirne blelbt der Mutter eines unehelichen Kindes so wenig erspart wie einem MHdchen in diesem Haus! ... Dem Weibe gewhhrte die Natur den Vorzug, mit seiner Liebe handeln zu kUnnen, deshalb mbchte die bulfgerliche Gesellschaft, die vom Manne regiert wird, diesen Handel immer und immer 
wieder gern als das §̧chmachvollste aller Verbrechen hinstellen! 13

Wedekind drilckt sich sehr klar aus: Durch die sexuelle Unterdrllckung des Weibes von der blirgerlichen Gesellschaft, die der Mann regiert, wird die Frau ihres natlrlichen Vorzugs beraubt. Die Prostitution 1st das gute Recht der Frauen! Von alldem inniglich bewegt, was Casti Piani ihr offenbart hat, will Frtulein Malchus ihn heiraten. Sie wird tber, menschliche Opfer fllr ihn aufbringen. Er erwidert: "Ihre Ubermenschlichen Opfer witrden mir im besten Falle die Eingeweide umkehren. Zeit meines Lebens liebte ich Tigerinnen. Bel Hllndinnen war ich immer ein Stlick Holz."14 Die Ergtnzung dieses Gedankens lenkt das Thema des Dialogs wieder auf die Prostituierte.

Wie wird die Welt des Freudenmtdchens sein, wenn sie thr Recht, sich zu verkaufen, erworben hat? In schwlirwerischen Zugen malt Casti Piani diese glunzende Zukunft:

...wie stolz steht das Weib in der Welt, sobald es das Recht erkampft hat, sich, ohne gebrandmarkt zu werden, zum hbchsten Preis, den der Mann ihm bietet, verkaufen zu kUnnen! Uneheliche Kinder sind bei der Mutter dann besser versorgt als die ehelichen beim Vater. Stolz und Ehrgeiz des Weibes sind dann nicht mehr der Mann, der thm seine Stellung anweist, sondern die Welt, in der es sich den holchsten Platz erkimpft, den sein Wert ihm ermbglicht. Welch herrlichen, lebens frischen Klang dann das Wort Freudenmidchen erhHlt. 15

Das utopische Muster eines zuklinftigen Staats steht uns plbtzIich gegentber. Worauf mag dieser Staat ruhen? Was dient als Pfeller? 
Als Einleitung zu diesem Thema gebraucht Wedekind eine rlhrende Erzthlung aus Castl Planls Jugend. Sle war so schrecklich, dass Fryulein Malchus aus tiefster Berlhrung wieder die Ehe vorschlHgt. Sie will thn liebkosen, aber er dringt sie zurldck mit der Ausserung, "Sie verstehen sich nicht auf Llebkosungen, mein Frdulein!" Es fehlt thr, melnt Casti Plan1, "...an dem nbtigen Zartgefth1 und Schamgeflh1..." Diese Geflhle besitzt jedes MHdchen in seinem Haus, glaubt er. Noch dazu besitzen seine MHdchen die "unschuldige Gltlckseligkeit und glllckselige Unschuld" in ihren Gesichtszigen. Diese Eigenschaften besitzt Fralulein Malchus auch nicht, deshalb kann sie nicht lieben. Zurllckgewiesen als Liebhaberin und Frau, gedenkt sie Prostituierte zu werden, denn sie ist vollkommen Uberzeugt von Casti Pianis Argumenten. Mit dem Ausruf: "Das wirde grauenvo11!" ${ }^{19}$ weist er Frtulein Malchus' Angebot zurldck. "Lassen Sie Ihre fllrchterliche Hand von dem einzigen gUttlichen Lichtstrahl, der die schauerliche Nacht unseres martervollen Erdendaseins durchdrHngt." 20 Die ehemalige Frauenrechtlerin versteht kein Wort davon und fragt, was dieser Lichtstrahl sein kbnne. Casti Plani erwidert:

Das ist der Sinnengenuss, mein gnddiges Frdulein! Der sonnige, lachende Sinnengenuss! Der Sinnengenuss ist der Lichtstrahl, die Himmelsblume, well er das einzige ungetrubte Gillck, die einzige reine, lautere Freude ist, die das Erdendasein uns bietet. Glauben Sie mir, dass mich seit einem halben Jahrhundert nichts mehr in dieser Welt zurlickhllit als die selbstlose Anbetung dieses einzigen aus voller Kehle auflachenden Gllickes, das im SInnengenuss den Mensch fur alle Qualen des Daselns entschldigt!21 
Der Pfeller selner utopischen Idee der Prostituierten ist also der Sinnengenuss. Auf dieses Fundament baut er sein ganzes Leben. Seine Uberzeugenden philosophischen Argument schelnen dem Fundament des Baus Stabilitat zu geben. Fralulein Malchus nort jemand kommen. Es aind Lisiska und ein Kunde, Herr Konig, der auch Wedekind darstellen soll. 22 Alles lst fur das Zwiachenspiel bereit.

Wle Tedekind schon sagte, wtrde er alle seine Anschauungen tber die Frauenemanzipation und die klufliche liebe im Lauf des Handelns widerrufen. Das Zwischensplel dient dazu. Bis jetzt lat alles in dem Schauspiel philosophisch-theoretisch gewesen. Argument, Erwiderung und logische Jberzeugung gaben uns unser Bild der Welt der Prostituierten. Jetzt wird das ganze philosophisches System auf die Probe gestellt. Frkulein Malchus und Casti Piani verstecken sich hinter einer Efeuwand, um Lisigka und Berrn KBnig zu belauschen.

Das ganze Zwischenspiel ist in Vers geschrieben. Die Szene beginnt mit einer Beschrelbung, die uns wohl bekennt sein durfte:

Ilsiaka in einfachem, bis zur Mitte der Wade reichenden, weissen Gewand, schwarzen Strtlmpfen, schwarzen Lackschuhen 23 ine weisse Schleife im offenen schwarzen Haar. 23

Ein Bild eines der Freudenmadchen aus dem Sonnenspektrum kommt uns in den Sinn. Wír merken aber, dass die Farben sich gethdert haben, oder vielmehr, dass Lisiska nur schwarz und weiss trkgt, die eine Farbe ist die Abwesenheit von allen Farben, die andere, eine Mischung von allen Parben. Die 
Farben der Sonne, des Lebens, sind verschwunden. Ob einige der freudigen sonnendurchdrungenen Ansichten des Sonnenspektrums tberlebt haben?

Herr $\mathrm{kHnig}$, ein Besucher des Hauses, tritt mit Lisiska, der Prostitulerten, auf. Wir sollten unsere Aufmerksamkeit auf den Namen Lisiska richten. Lisiska war der Deckname der Frau des rbmischen Kalsers Claudius. Ihr wirklicher Name war Messalina und Jeden Abend schlich sie zum Bordell, um ihren krankhaften Trieb zu befriedigen. 'Heutzutage wllrde man sie als Nymphomanin bezelchnen. Wedekind hat den Namen Lisiska mit Sorgfalt gewthit.

Der Dialog in Vers beginnt mit einer sachlichen Kusserung von Herrn KBnIg:

Ich kome nicht, die Zeit mir zu vertreiben

Als Wolltsting in delner Reize Bann, Und will dir dankbar und gewogen bleiben, Wenn bald arnllahtert Ich von hinnen kann.25

Die unerwartete Antwort weist seine Freundlichkelt zurllck und verlangt ganz etwas anders:

HHufen Sie mitleidlos Qual auf Qual! Wenn Thre Faust mein Gesicht zerschiluge, WHr's meiner Sehnsucht noch kein Genuge.'26

Herr KOnig versteht sie nicht. Er erwidert verzweifelt: "Du sprichst, als blisstest du im Fegefeuer/Schon hier die Strafen fur genossne Lust." 27 Lisiska antwortet:

Im Gegentel1! Die Lust, das Ungeheuer, Tobt ewig ungezthmt in dieser Brust!

Meinen Sie, ich Teufelsbraten whre je in dies Haus geraten Wenn von des Herzens grässlichem Klopfen

Freude mich kbnnte befreien? 
Freude zerstiebt, ein Tropfen

Auf heissem Stein!

Und die Wollust, unstilt,

Ein hungerndes Jammerbild,

Sturzt sich, dass sie den Tod finde,

In alle Abgrtinde! - - 28

Nur ungeatillte Wollust trieb Lisiska ins Freudenhaus; die

Freude lat zu schwach, sie von ihrem krankhaften Trieb zu befreien. Casti Pianis "sonniger, lachender Sinnengenuss" scheint irgendwo anders zu Hause zu sein, aber nicht hier. Hier tobt die ewige unzthmbare Iust in der Brast dieses sogenannten "Freudenmadchens".

Herr Konig staunt, denn er hat Lisiska besonders aus der Schar Madchen auggewahlt, nur weil "ein Strahl unschuldiger Gllickseligkeit" aus inren Augen leuchtete. 29 Dies, nach Cest1 Piani, sel eine Eigenschaft, die eine Frau, die fur den Iiebesmarkt geschaffen ist, ausotrahlt. 30 Scheinbar haben Oesti Piani und Herr Konig(Wedekind) des leuohtende Element in Iisiskas Augen volilg verkannt. Die ungestillte Iust und nicht die unschuldige Glthckseligkeit ist es, die so lockend aus den Augen scheint.

Lisiska wiederholt thren wunsch, geschlagen zu werden. Da Herr Konig ihr Verlangen nicht stillen mbchte und sogar das Haus verlassen will, weint sie inn weiter in die Tiefe ihres Verlangens ein, in der Hoffnung, etwas Mitleid in thm zu erwecken. Voll Verzweiflung fthrt sie fort:

Wenden Sie sich nicht herzlos ab!

Vor mir hab ich mein Grab

Und hoffe nur noch, aus dieser Welt

Moglichst viel mit hinabzunehmen.

Glauben Sie, solche Begierden ktmen, 
Weil dies Haus uns gefangen hHlt?

Nein! Nur der Sinne folternde Gier

Bannt uns hier!

... Nacht fllr Nacht

Seh ich es blendend sonnenklar,

Dass selbst in diesem Haus kein Frieden

Den Sinnen beschieden.31

Von threm Versteck hbren dies die beiden Lauschenden. Ihre Reaktionen auf die Wahrheit, d.h, auf die nackte untheoretische WirkIIchkeit lautet:

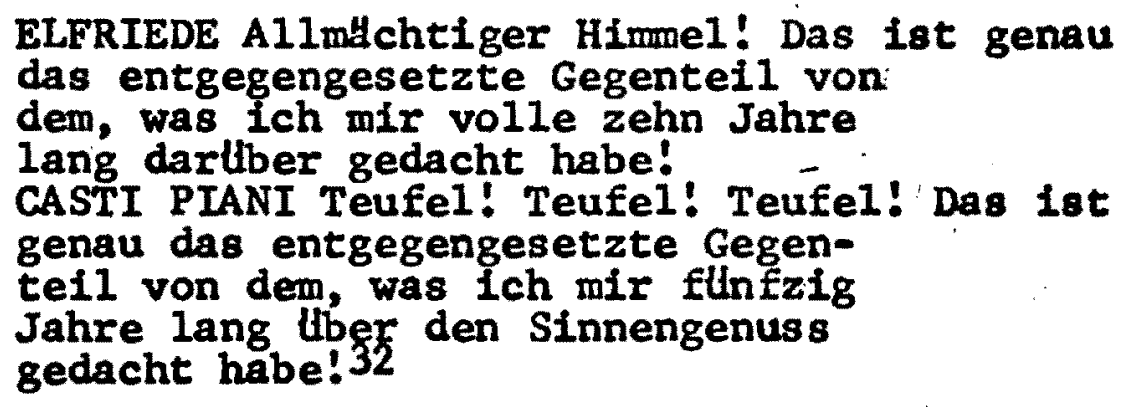

Lisiska erzthlt Herrn Kbnig die Geschichte ihres Lebens und wie sie die Freude in der Liebe suchte. Doch dies wurde ihr nie gewthrt, "denn es war stets nur der hbllische Trieb,/ Aus dem an Freude nichts thrig blieb." ${ }^{33}$ Ihr Ubersteigertes sexuelles Verlangen siegte immer ther das Freudengefthl, bis das Verlangen nur durch Gelsseln zum Teil gestillt werden konnte. Ihr nymphomanischer Trieb ist schuld an ihrem Leben im Freudenhaus. Die scheinbar kryptische Bemerkung ther die Unschuld, die Herr kbnig jetzt macht, wird durch Lisiskas Ausserung tber den h811ischen Trieb geklHrt:

Der Unschuld welsses Kleid trägst du. Dir hat Selbst dieses Haus die Seele nicht geschändet. Von deiner Reinheit ist mein Aug geblendet, An deinem Bild sieht sich mein Herz nicht satt. 34

Thre Unschuld ist ihr geblieben, weil ihr abnormaler Trieb sie gezwungen hat, Erlbsung in einem Freudenhaus zu suchen. 
Wie eine heilige Martyrerin wird sie dem Trieb unterliegen. Wie eine Heilige muss sie die Schmerzen ihrer Qual auf Erden bektmpfen. Herr Kønig beschreibt Lisiskas Kampf und seinen Entschluss:

Im Selbstmord schwelgend ohne Unterlass, Kumpfit du mit nie erforschten Seelenschmerzen, Den Tod im Antlitz und den heissen Hass Auf alles eitle Erdengluck im Herzen! ... In keuscher Dichtung soll durch mich die Welt Verkaufter Liebe ermessen lernen. 35

Dies hat Wedekind getan. Er hat uns die Geschichte der Prostitution durch Lisiskas und Herrn Konig erzlhlt. Nur der letzte Peitschenhieb fehlt noch. Iisiska sorgt flur ihn:

HERR KONIG Bist du aus Liebe jemals froh geworden?

IISISKA Wenn Sie jetzt gleich mich ermorden,

KUnnt meine Rede nicht anders sein.

Immer nur war es der hollische Trieb, Aus dem an Freude nichts thrig blieb...

So ist's in diesem Haus nun einmal:

Alle begegnen sich hier,

Denen die Ilebe unendliohe Qual

Und niegestilite Begier. 36

Die lange Reise in den Tod wird ftr die beiden mit Lisiskas Worten bereitet:

Mir lat als hychste Wollust langat ein Land Orew'ger niegestyrter Ruh bekannt.--

Ach, dass ich unter deinen Flusten sturbe 137

Was mag Fraulein Malchus' und Cast1 Pianis Reaktion auf diese rernichtende Offenbarung sein? Schon in der Mitte des Zwischenspiels erfuhren w1r, dass die beiden das Gegenteil ihrer Ansichten tber den Sinnengenuss in Lisiskas Ausserungen sahen. Jetzt mllssen sie sich damit abfinden. Casti Piani spricht rerstort ror sich hin:

Was tu ich noch auf der Welt, wenn auch der 
Sinnengenuss nichts als hbllische Menschenschllthterel 1st, wie das ganze Ubrige Erdendaseln!38

Frtulein Malchus ist hingerissen von Lisiskas Botschaft:

Was habe ich nichtswlirdige Schmarotzerin mir

in meinem vertrockneten Hirn unter Sinnengenuss

vorgestellt? :--Selbstaufopferung, glthendeg

MHrtyrertum ist das Leben in diesem Haus.39

Cast1 Pian1 sucht einen Ausweg und Frtulein Malchus glaubt schon einen gefunden zu haben: Sie will Prostituierte werden und Casti Planl soll sie verkaufen. "Das hatte gefehlt!", sagt der MHdchenhHndler und erschiesst sich. Sein letztes Wort lautet:

So!--So, so, so!--Ich--ich habe euch -betrogen! --Der Sinnengenuss--Menschenquallerel --Menschenschinderei-.--.---endlich--end11ch-Er1bsung!41

"Und was hat thn 80 zerschmettert? ihn, den Propheten der Fleischeslust? Der viermalige Jammer des Freudembldchens Llsiska: "Immer nur war es der hblilsche Trieb,/aus dem an Freude nichts tibrig blieb...."

Fraulein Malchus drilckt seine Augen mit den folgenden Worten zu:

Die letzte EnttHuschung hast du dir noch wohl in deinen furchtbarsten Weltschmerzen nicht trdumen lassen, dass dir eine Jungfrau die Augen zudrilickt!--Darauf drthckt sie ihm die Augen zu und sinkt jammervoll weinend zu seinen Flis sen. 43

Tod und Teufel und Insbesondere das Zwischenspiel befasst sich wohl mit dem Thema der Liebe, aber was fllr eine Art Llebe: Es scheint, als ob der Kult der Astarte in seiner ganzen Grausamkeit sich hier zu Hause flhit. "Hier lst das 
hbilische Spiel und Widerspiel der Liebe als Leiden und Leidenmachen aus Wollust an einen wie am anderen mit Schthrfe Im Relief herausgetrieben." 44 Der nat beinah griechischen Tragbdie 1st der Tod. Die ungezthmte, ungezligelte Sinnlichkeit flhrt auf keinen Fall zu einem 1dyilIschen Dasein auf Erden, wie Wedekind-Casti Plani dachte. Der Tod steht triumphierend vor Wedekinds Welt der Sinnlichkeit und ihrer Trkgerin, der Prostituierten. Er muss einen Ausweg aus diesem tbdilchen Dilema finden, 'wenn sein Begriff der Sinnlichkeit gerettet werden soll. In seinem nllchsten Werk Uber die Prostituierte Schloss Wetterstein werden wir solch einen Versuch sehen. 
RAPITEL V

SCHLOSS WETTERSTEIN

Im Jahre 1910 exschienen die drei Telle des Schauspiels Schloss Wetterstein als Einakter. Die drel Titel sind: In allen Sutteln gerecht: Kombdie in elnem Aufzug, Mit allen Hunden gehetzt: Schauspiel in einem Aufzug und In allen Wassern gewaschen: Tragbdie in einem Aufzug. Da sie zu verschledenen Zeiten in diesem Jahre erschienen, wurden sie erst nach dem Erscheinen des letzteren von Wedekind zu einer Einhelt zusamengefasst.

Der Autor versucht in diesem Drama einen Ausweg aus dem Dilemma, das thm soviel Sorge in Tod und Teufel bereitete. In seinen Worten: "Wle fltr die Ehe, so versuchte ich in dem Drama Schloss Wetterstein auch fllr die Prostitution eine absolute Formel zu finden, die meines Wissens noch nicht existiert." 1 Das Drams wird von ihm als eine Schilderung der Familie und deren Gegensatz, die Prostitution, gesehen.' Die ersten zwel Akte beschHftigen sich mit der Ehe und Familie. Der dritte Akt, "In allen Wassern gewaschen", den wix hier behandeln wollen, beschtftigt sich mit dem Kind dieser Ehe, Effie.

Sie ist Prostituierte und wohnt als "Edelhure" 4 in dem Schlosse Wetterstein, das einst Ihrem Vater Rlldiger Wetterstein gehbrt hatte. Ein PrInz sorgt fur ihren Unterhalt. 
Sie ist von Pracht und milrchenhafter Schbnheit umgeben. Ein Prinz, ein mürchenhaftes Schloss, eine Edelhure: mit diesen drel Elementen kann das fantastische aussernatlirliche Schausplel beginnen. Ob Wedekind die Lbsung zum Problem des Sinnengenusses in diesem "...Ubergang aus der Realitlt ins Mythische..." findet, werden wir sptter sehen. Sicher 1st, dass er die LUsung in der Welt der Wirklichkeit nicht fand. Der dritte Akt ist gänzlich in Jamben geschrieben, die den abstrakten Charakter des Schauspiels noch deutlicher machen.

Der erste Auftritt beginnt mit Professor Scharlachs Bemerkung zu Heiri Wipf, dem GHrtner, dass das Schlossfrlulein, Effie, sich nicht wohl fthle. Effie selbst erscheint jetzt, um uns ihre Krankhelt und deren Folgen zu er1Hutern. Heulend weiht sie uns ein:

Mein Stolz, mein tbermut, mein Zeitvertreib, Mein Abenteuerleben, meine Freiheit, Ich dankte alles meiner Weltanschauung. Von jedem Blick, der einen Menschen einfing, Von jeder Nacht, die ich durchtobt, habe ich Gewissenhaft mir Rechenschaft gegeben, Nicht anders, als wenn anvertrautes Gut Ich zu verwalten hutte. Und nun hbr $1 \mathrm{ch}$, Dass alles Krankheit, mein Verlangen Krankheit, Die Augen Krankheit, meine Farbe Krankheit! 6

Durch eine medizinische Erklurung wird es Effle klar, dass ihre Sinnlichkeit, die sie zur "Weltanschauung" erhoben hatte, nicht urwilchsig, sondern erst durch die Krankheit entstanden ist. Ihr Verlangen ist die Folge elner Verdauungsstbrung, ihren Augenschimmer verdankt sie elnem Leberleiden, und thre Hautfarbe und deren Mattigkeit wird von Gallensteinen 
verursacht. Effie steht vor der bedeutendsten Entscheidung Ihres Lebens: wie, oder ob sie den Verlust ihrer Weltanschauung Uberstehen kann, oder will. Sie sagt:

Erloschen ist des Lebens Flammenpracht, Nur freudlos dustre Kohlen bleiben tbrig. Verkaufs-Entsthnung, Dirnen-Heiligung, Wie albern, wie entsetzlich schal mir das Jetzt klingt! Und doch kehr ich nicht um. Jetzt kommen

Verfall und Niedergang. Sie sollen aufrecht Und stolz mich finden, wie das Gillck mich sah. ${ }^{9}$

Hier haben wir es mit einer ungewbhnlich starken Persinlichkeit zu tun! Effie erinnert nicht an die MHdchenhaftigkeit der Ella oder die freudige sonnige Sinnlichkeit der Sonnenspektrumbldchen, und weit weniger erinnert sie an die krankhaft dunkle Gestalt Lisiska in Tod und Teufel; Effie ist selbstbewusst und, obwohl sie auch dem Tod entgegengeht, entscheidet sie mit geistiger Klarheit und Besonnenheit ihr eigenes Schicksal. Sie wird nicht getrieben, sie geht freiwillig und stolz ihrem Schicksal entgegen. Mit Effies sachlich gesprochenen Worten verkindet sie einen Kampf, denn sie muss thren Beruf weiterhin unter schwerer gewordenen Bedingungen austlben.

Karl Salzmann, Effies Manager, tritt auf und teilt uns mit, dass Mr. Chagnaral Tschamper aus Atakama bald auf Schloss Wetterstein eintreffen wird, un Effie hunderttausend Dollar fllr thre Gunst zu bezahlen. Er hat nux einen Wunsch: in den Axmen eines MHdchens seinen Tod zu finden. Effie kann seine Ankunft kaum erwarten, denn mit Tschamper kommt der Kampf, der sie "aufrecht und stolz" niedergehen lassen soll. 
Sie welss, dass ihr Verfall sicherlich kommt, aber sie wird dennoch kampfen.

Im nHichsten Auftritt ruht sich Effle vor dem Kampf aus. Sie unterhtlt sich mit Salzmann, ihrer Mutter Leonore, Ihrem Vater Rlldiger, mit Uhlhorst, dem Anarchisten, mit Taubert und Schigabek, dem Philosophen und dem Troubadour und mit ihrem Leibarzt, Dr. Scharlach. Im Laufe der Unterhaltung erzthlt Effie von Ihrem Liebhaber, dem Prinzen und wie sie ihm ihre Weltanschauung Uber die Sinnlichkeit beigebracht hat. Sie erldutert ihre Philosophie der Sinnlichkeit in dem folgenden Dialog:

TAUBERT Nur unsre Liebesstufenleiter hast Du deinem Prinzen schwerlich schon/ verraten!

EFFIE Die Liebesstufenleiter lallt er manch-/ mal

Schon tief im Traum. Als im Torpedohafen Schiffotaufe war und er die Rede, die man Ihm In die Hand gedrllckt, nicht lesen/ konnte, Da sagt' ex, ohne lang sich zu besinnen: Erstens im Dunkeln, zweitens im Lampen-/ schein-Sklavische Brut in veryngstigter Pein!

UHLHORST Drittens beim Tageslicht, viertens im/ Freien--

Freuden, die wir auch im Tod nicht/ bereuen?

SALZMANN Funftens in Nacktheit, sechstens vor/ Spiegeln--

Hei, wie in Sturm sich die Sinne be-/ flügeln!

TAUBERT Slebtens im Perlenschmuck, achtens im/ Festgewand--

Lungst sind die Slinden der Knechtschaft/ verbannt!

EFF IE Neuntens im Wettkampf, zehntens als Opfer-/

Das unsre Gottheit uns nicht mehr veribsst! 10

So lautet ihre Philosophie der Liebe. Schon im zehnten 
Gebot dieser Liebe ahnen wir Effies Schicksal: "...zehntens als Opferfest". Wer wird das Opfer sein, sie oder Tschamper? Mr. Tschamper kommt an und alle ellen thm zu. Nur Taubert, der Philosoph, bleibt zurllck, um Effle vor Tschamper zu warnen, denn er habe schon drel oder vier Freudenmbdchen umgebracht. Selbst wenn sie zurlkktreten oder absagen wollte, k\&nnte sie nicht, meint sie, denn der Prinz braucht Geld, Ihre Eltern brauchen Geld und Salzmann bekommt auch hunderttausend, wenn sie Tschampers Bedingung erfllit. Kurz: Sie lst verpflichtet. Diese Grilnde sind nicht die einzigen, noch zwel weitere liegen vor. Effie spricht:

Mein Leben ist mir elnfach nicht ertrlgilch, Wenn nicht ein Abenteuer mich erwartet,

Bel dem kein Mensch den Ausgang ahnen kann.11

Dies genlgt Taubert nicht und Effie ist bereit, die Wahrheit zu 8agen: "Qull mich nicht IHnger! Ohne Llebe kann Ich/Nicht leben." Tschamper zu empfangen, aber ihr sinnliches Verlangen dient als Hauptgrund dazu.

Zu TrHnen durch Tauberts Befragen gerelzt, vergisst Effie thre Freude momentan Uber Tschampers Ankunft. Salzmann tritt auf und ermahnt sie zur freudenmddchenhaftigen Haltung, denn mit TrHnen verdient man wenig. Tauberts Beflichtung scheint thm selber jetzt lacherlich und Salzmann fllgt hinzu: "Beflurchtung? Wenn in allen Wassern man/Gewaschen 1st, dann hat man nichts zu flurchten!" ${ }^{13}$ Mister Tschamper aus Atakama tritt eln. Der Kampf kann beginnen!

Die Handlung des siebten Auftritts schreitet rasch fort. 
Tschamper macht klar, dass er gekommen ist, um den Tod zu suchen. Effie, da er sein Ziel so schnell wie nur mbglich erreichen mbchte, will sich sofort entkleiden. Dies erlaubt er nicht. Schon ist sie in threr Individualitut eingeengt, denn thre Kleldung verbirgt ihre sexuellen Reize, die ihrem Ich als Hussere HUlle dienen. Tschamper gewinnt die erste Runde.

Die Unterhaltung wendet sich zum Thema der Ehe. Effie aber mbchte auch schnell zum ziel kommen. Sie bletet sich nochmal an:

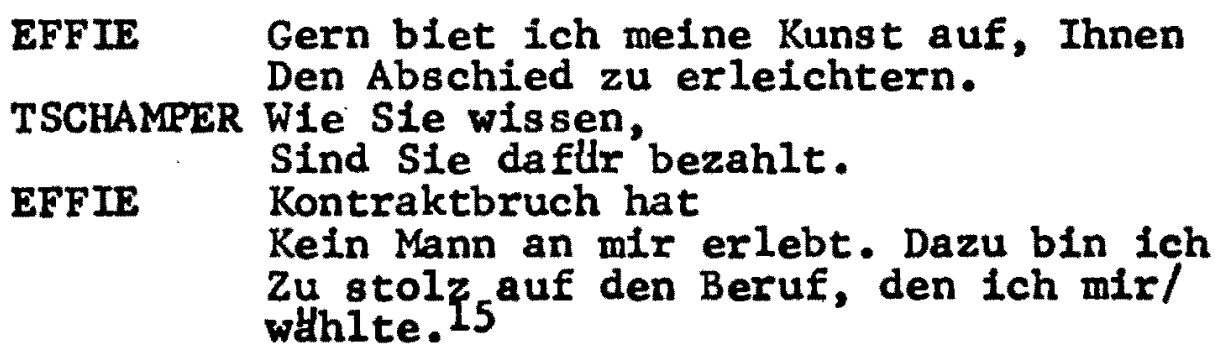

Nochmal Hussert sie den Wunsch sich zu entkleiden mit den Worten :

Und Sie verwehren mir, mich zu entkleiden?

Das ist nicht ritterlich. Ein Freudenmidchen,

In seiner Eitelkeit verletzt, ist hilflos,

Ist dumm und plump und bibde wie ein Kind.

TSCHAMPER So hilflos brauch ich Sie.16

Mit diesen Worten giesst er einige Tropfen Blaushure in Effies goldenen Pokal, den sie von dem Prinzen geschenkt bekommen hatte. Tschamper will, dass sie hilflos 1st, welss aber nicht, wie er das anstellen soll.

Da Effle thre Erregtheit kaum noch blundigen kann, versucht sie Tschamper durch die Erzhhlung von der "Zehnstufenleiter der Llebe" für sich zu gewinnen. Nur der neunte 
Interessiert ihn: "Neuntens im Wettkampf...." Er erwidert darauf:

Im Wettkampf! Herrlich! Ganz mein Fall! Nun aber Statt des Gedichtes Wirklichkeit! Erzhij mix

Das traurigste Erelgnis deines Lebens!ly

Mit dieser Ausserung beginnt der Kampf ernst zu sein. Sinnlichkeit als kHrperliche Eigenschaft interessiert Tschamper kaum. Sein Sleg tber Effle wird kein leerer sexueller Sieg sein, sondern ein Sieg des Geistes. Effle weigert sich, Tschamper etwas zu erzlhlen: "Nle hab ich den Beruf, Entsetzen zu/Vermitteln, mir erwhltt. Der Freude dien ich!" 18 Tschamper welss, dass er sle jetzt in seiner Macht hat, denn sie sieht eine Erzbhlung thres traurigsten Ereignisses als entsetzlich und zittert an allen Gliedern.

Zitternd erzlhlt die Edelhure die Geschichte threr traurigen the und wie ihr Mann sich an ihrer Brust erschossen hatte. Dies imponiert Tschamper nicht:

Das hat dich nicht drei Tage lang erregt. Wie 8011 ich mich zum Selbstmord daran gttrken? Herzt女ne will ich! Leben deine Eltern? 19

Vor Schreck auffahrend lehnt Effie Tschampers Verlangen $a b$, die Geschichte vom Tode ihres Vaters zu erzthlen. Zum vorletzten Mal versucht sie ihn zu verflhren. Sie fllit ihm um den Hals mit threr Bitte:

Komm mit mir! Bleiben wir nicht hier.

Du fragst nachher nach meines Vaters Tod

So wenig wie nach meines Gatten Selbstmord. 20

Er schlagt ihre verzweifelte Bitte $a b$ und steigert ihre Hilfslosigkeit aufs Hلchste. Jetzt ist sie endglltig entwaffnet; Ihre Seele liegt nackt vor dem MilliardHr aus Atakama. Der 
Sieg droht.

Effies Verflhrungsversuch ist fehlgeschlagen. Nun muss sie Tschamper erzthlen, wie ihr Vater starb. In steigender Erregung werden die Folgen des Duells, in welchem ihr Vater ums Leben gekommen ist, geschildert. Tschamper fragt nach threr frlihsten Erinnerung an ihre Eltern. Seine psychoanalytische Methode hat den erwarteten Erfolg: Sie spricht ohne Pause. Noch gespannter, noch gesteigerter wirbeln Effies Erinnerungen un sie, bis T'schampers sorgfHltig gestellte Frage sie vbliig hilflos macht: "Erinnerst du dich deiner Eltern noch,/Wie sie sich schalten, schlugen, sich verfluchten?" ${ }^{21}$ Diese Andeutung auf thre Eltern bringt den folgenden Dialog zustande:

\begin{tabular}{|c|c|}
\hline TSCHAMPER & 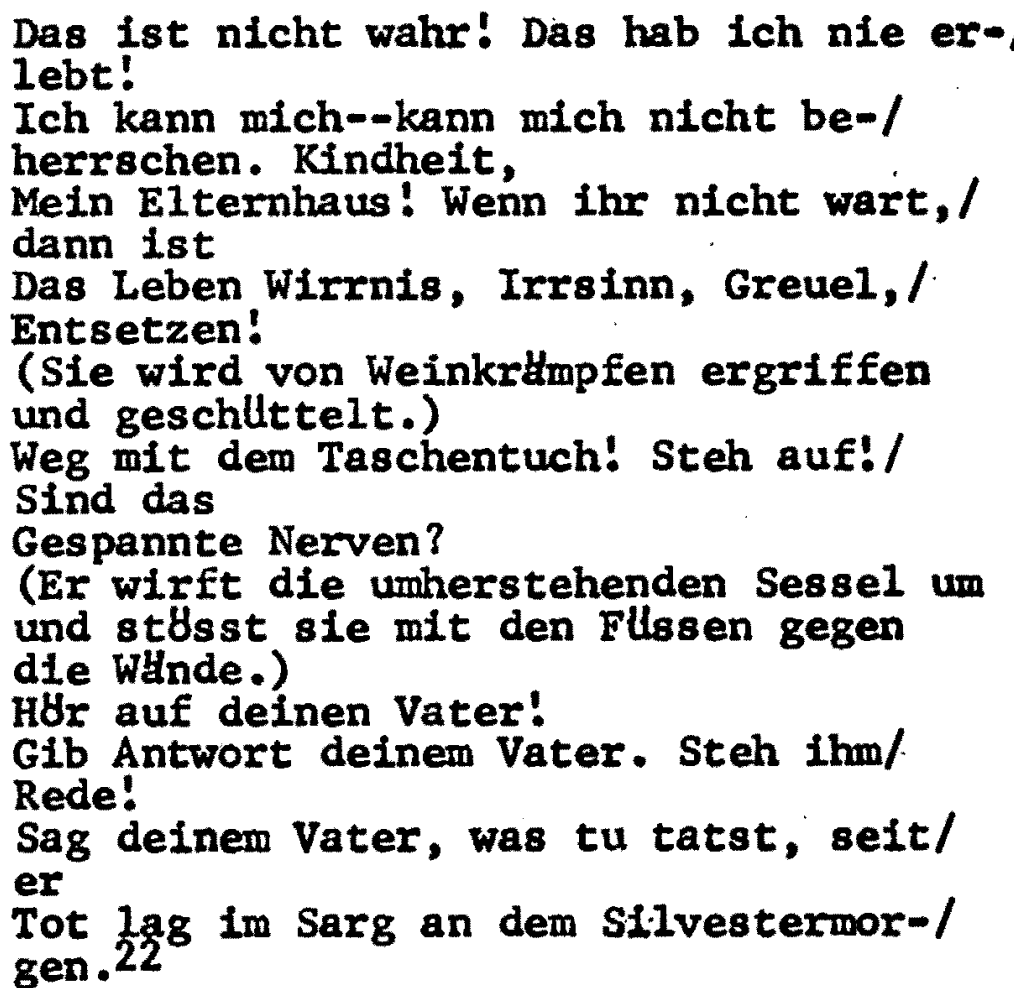 \\
\hline
\end{tabular}

Sie versucht zum letzten Mal inn zu verflthren, aber dieses Mal handelt sle aus neuentdeckter Liebe zu ihm, und zwar eine 
geistige, kdrperlose Liebe. Ihre therwindung der kbrperlichen Liebe hat gleich Folgen. Ihr Ende ist gekommen, wie folgender Dialog zeigt:

EFFIE Nimm dich, Geliebter, deines Lebens/ an.

Bis heute hast du nicht gelebt.

TSCHAMPER Auch du nicht.

EFFIE Nie ward ich Dirne, hltte mich ein/ Bundiger Wie du gebHndigt.

TSCHAMPER Hast du mich gesucht?

Ein Mensch, der Mut hat, sucht sich/ seinen Bundiger.

EFTIE Dir fehlt das Weib, das álles fur/ dich opfert. Mir fehlt der Mann, dem ich mich/ opfern darf.

TSCHAMPER Und du, mein Kind, glaubst dieses/ Weib zu sein?

EFFIE Bel meinem Stolz, wenn du noch da-/ ran zweifelst, Dann trink Ich diessen Trank.

TSCHAMPER Ich zweifle dran.23

Mit diesen Worten greift Effie nach dem Giftbecher und trinkt. Tschamper schaut entzlickt zu und spricht:

Wie sonderbar, dass nie sich eine Dirne,

Wie's doch bei Kindern allgemein sonst lblich, tber ihr Elternhaus erhaben fllthlt:--

Wie dir ergeht's noch vielen.24

Was Effie, die stolze Edelhure, getdtet hat, kann aus der Handlung herausgelesen werden. Wedekinds "absolute Formel" fur die Prostitution und Ihre Vertreterin wird von Rothe formuliert:

Sobald die Dirne gezwungen wird, sich an den individuellen Ursprung, an ihre Kindheit im Elternhaus zu erinnern und den Bereich des Sexuellen zu verlassen, soll sie gegentber der patriarchalischen Forderung nach selbstloser Unterordnung der Frau 25 gegen die sie sich auf-

Im wahrsten Sinne des Wortes war Effie hilflos und die Hilf- 
1osigkeit vor einer Urgewalt, wie Tschamper, ist total. Es 1st wahr, dass Effie Tschamper unterliegt, aber keineswegs wahr, dass sie gebrochen untergeht; sie geht aufrecht und "...siegend ihre schbne Kraft freispendend, verschwendend", zu Grunde.

Die Prostituierte in Schloss Wetterstein siegt Uber den Sinnengenuss, bezahlt aber mit ihrem Leben. Im Sonnenspektrum ahnten wir die tbdlichen Folgen des ungeztigelten Sinnengenusses. In Tod und Teufel sahen wir deutlich, dass Lisiska durch Ubersteigerte Sinnlichkeit stirbt. Schloss Wetterstein zeigt uns eigentlich keinen Ausweg aus diesem Dilemma. Die Sinnlichkeit als Grundstein einer sexuellen Weltanschauung bietet nur den Tod als Belohnung. Einen stolzen Tod hat Effie gefunden, aber doch den nackten Tod. Als Epitaph flir Effie gelten folgende Worte:

Im Embryo oder im verwesenden Kadaver, im $8 \mathrm{chwel1-}$ enden Phallus wie in den letzten Zuckungen der Sterbenden vermag der Mensch erschreckend die gestaltlose Fremdheit seiner Ursprilnge und Ziele ahnen; und die Erkenntnis, dass er nur im Geformten lebt, ist der Grund fur die von jeher strenge Tabuierung dieser Bezirke. Sucht man aber in ihnen das "elgentliche" Leben auf, so wird man digr entfesselten Wut der Chimiren nicht entgehen. 27

Effie, die Edelhure, ist thnen nicht entgangen. 
KAPITEL VI

SCHLUSS

Zwischen Elins Erweckung (1887) und Schlo8s Wetterstein (1910) liegt eine Zeitspanne von 23 Jahren. Whhrend dieser Zeit entwickelte Wedekind seine Idee der Prostituierten zu seinem eigentlichen Ausgangspunkt in Schloss Wetterstein. Zwar schrieb er weiterhin Dramen, aber nie wieder befasste er sich leidenschaftilich mit dem Freudenmldchen. Nie wieder trat sie als Hauptfigur auf, denn andere Probleme wle Schamgefthl und Elfersucht (SImson, 1913) oder sein lebhaftes Interesse an dem Mephisto-Faust Problem (Franziska, 1911) schienen inm wichtiger.

Man versucht aus den mannigfaltigen Erfahrungen und Impressionen, die Wedekinds vie $x$ "Prostitutionsdramen" einem erteilt haben, irgendeine Formel fur die Prostitution zu gewinnen. Vlelleicht dient Ernst Schweizers Bemerkung ther den Gehalt von Wedekinds Werk dazu: "Genuss wird Opfer, Lust Vernichtung." In den drei spüteren Dramen sahen wir dieses Prinzip deutilch funktionieren. Das Sonnenspektrum zeigte uns wohl frohe begehrenswerte Freudenmildchen, aber stHndig Im Hintergrund wurden wir an Minetta, die sterbende Nymphomanin, erinnert, deren Lust Vernichtung wurde. Tod und Teufel schilderte LIsiska, auch eine Nymphomanin, die ihrem "hb1li- 
schen Trieb" zum Opfer fHIIt. Niemals wurde sie aus Liebe froh, niemals war Ihre Lust etwas anders als elne Rutschbahn zur Vernichtung. Und zuletzt haben wir Effie, die Edelhure, eine Art Super-Prostituierte. Sle scheint einen gelstigen Sleg ther die vernichtenden Gesichtspunkte der Sinnlichkeit zu felern, in der Welt der Wirklichkelt, jedoch stirbt sie an Gift; Opfer und Vernichtung sind Worte, die Ihren Untergang beschreiben. Die Sinnlichkeit war in jedem Fall daran Schuld.

Obwohl der Ausgangspunkt in Bezlehung auf Lust und Vernichtung in den Dramen thnlich 1st, wtre es ein Fehler, Wedekinds hier behandelte Dramen nur von diesem Standpunkt aus zu sehen. Wir merkten in jedem Stllck, dass Wedekind nicht selten zur Tendenzdichtung neigt. Dieser Punkt ist ebenfalls wicht1g, wenn wir seine Prostltulerte verstehen wollen.

Jedes Drama, das behandelt wurde, zelgt Neigungen zur Tendenzdichtung. Elins Erweckung wimelt von Bemerkungen gegen die blirgerliche Gesellschaft In Beziehung zur Prost1tulerten. Sonnenspektrum macht manche Aussage gegen die sexuelle Erziehung in der butrgerlichen Gesellschaft. Tod und Teufel zeigt die bifgerliche Gesellschaft als verantwortlich fllr den schlechten Ruf der Prostituierten. Weniger tendenzneigend ist Schloss Wetterstein, aber auch dies Drama sieht die Prostituierte als wesentlich von der sozialen Struktur der Familie beeinflusst. Wedekinds Dramen sind nicht unmittelbar sozlal wie die von Hebbel oder Hauptmann, "...sie 
gehen nicht auf diese oder jene soziale Seite, sie gehen auf das Ganze der menschlichen Gesellschaft". Seine Dramen sind also tendenzibs in einem weiteren fast allgemeinen Sinn. Wedekinds Prostituierte sind nie reine Sozialprodukte, obwohl die Gesellschaft eine grosse Rolle In ihren Leben spielt. Sie sind Freudenmbdchen entweder aus Nelgung oder aus physischen Gritnden. Alle seine Prostituierten sind Produkte seiner Liebe, alle zeigen in ihren sorgftltigen philosophischen Behandlungen, wie sie von ihrem Autor geliebt werden.

Die Liebe zur Prostituierten treibt Wedekinds Schilderungen bis zum Hussersten Rand der RealitHt--von Ella bis zu Effie. Viele wollen elne Verherrlichung der zugellosen Sinnlichkeit in seiner Gesamtvorstellung der Dirne sehen. Dies ist weit von der Wahrheit entfernt. Nicht nur sollen seine Dramen keine Verlockung sein, sie sollen eine Abschreckung von der allzufreien Sinnlichkeit aufgefasst werden.

\section{Abschied}

Die Sinnlichkeit gibt mir Abschiedsfest;

Das sind kuriose Gestalten,

In Binden gewickelt, in Schienen gepresst,

Und kaum mehr festzuhalten.

Die strahlende Nacktheit such ich so bang,

Es fehlt thr wohl an Vertrauen.

Ich hab sie bel gellendem Becherklang

Zu haufig zusammengehauen.

Und ist erst das Seelenleben entweibt, Dann sind sumtliche Lampen erloschen. Fur das, was flir mich dann noch tbrig blelbt, Daflur gebe ich nicht einen Groschen.5 


\section{ANMERKUNGEN}

\section{KAPITEL I}

\section{1}

Frank Wedekind, "Konfession", Frank Wedekind: Prosa,

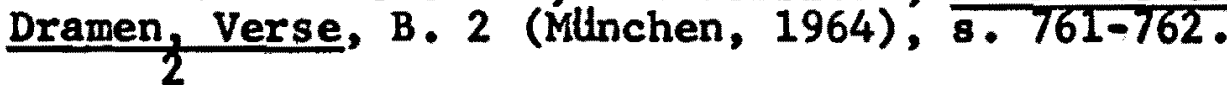
8. 10 .

Joachim Friedenthal, Das Wedekindbuch (Mlinchen, 1914), 3

Arthur Kutscher, Frank Wedekind und das Theater, Dre1 Masken Verlag, ed. (Berlin, 1915), 6. 34 . 4

Paul Fechter, Frank Wedekind: Der Mensch und sein Werk (Jena, 1920), 8. 21 .

5

Paul Fechter, Geschichte der deutschen Literatur, B. 1 (Gliters1oh, 1960), s. 366 . 6 Frank Wedekind,
(Mlinchen, 1924), s. 384 . 7 8 ebd., 8. 385 .

WIlhelm Bolze, Frank Wedekind und das Theater, Dre1 Masken Verlag, ed. (Berín, 1915), s. 74 . 9

Stefan Zweig, "Wedekind der Unblirgerliche.", Das Wedekindbuch, ed. Joachim Friedenthal (Mlinchen, 1914), 8. 242-243. 10 11

Fritz Dehnow, Frank Wedekind (Leipzig, 1922), 8. 52.

Lion Feuchtwanger, "Frank Wedekind", Neue Deutsche Lit-

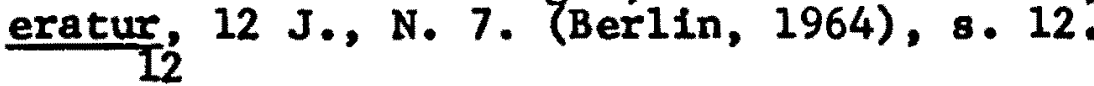

\section{3}

ebd., $8,12-13$.

14

Dehnow, 8. 52 .

Frank Wedekind, "Bltrgerliche Gesellschaft", Frank Wedekind: Selbstdarstellung, ed. Will1 Reich (MUnchen, 1954), 8 . 69. 15 16 Dehnow, 8. 52 .

Franz Ble1, Das Wedekindbuch, ed. Joachim Friedenthal (Mlinchen, 1914) 8. 141.

17

Friedentha1, 8. 47. 
1

Frank Wedekind, "Elins Exweckung", Frank Wedekind: Gesammelte Werke, ed. Joachim Friedenthai (Mlnchen, 1924), 8 . 50 .

2

ebd., 8. 51 .

3

ebd., 8. 51 .

4

Friedrich Rothe, Frank Wedekinds Dramen: Jugendsti1 und Lebensphilosophie (stuttgart, 1968), 8. 45 .

5

Wedekind, 1924, 8. 51 .

6

ebd., 8. 52

7

ebd., 8. 52

8

ebd., 8. 52 .

Hans Hellwig, Frank Wedekinds dichterische Anfunge

(Diss. Giessen, 1928), 8. 28 .

10

Wedekind, $1924,8,53$.

11

12

ebd., 8. 54 .

13

ebd., 8.54 .

14

ebd., 8. 55 .

15

ebd., 8. 55 .

L. R. Shaw, "Bekenntnis und Erkenntnis in Wedekinds

Die Zensur", Frank Wedekind: zum 100. Geburtstag, ed. Stadtbibliothek Mllnchen (Mlinchen, 1964), 8. 33 .

16

17

Wedekind, $1924,8.56$.

18

Wedekind, $1924,8.56$.

Frank Wedekind, "Kunst und Sittlichkeit", Frank Wedekind: Prosa, Dramen, Verse, B. 1 (Mllnchen, kein Datum), 8. 933. 19 20

Wedekind, 1924, 8. 61 .

21

Hellwig, 8. 17.

22

Wedekind, 1924, 8. 63.

Hellwig, $8,30$. 
23 24

Wedekind, $1924,8.64$.

1964) $25^{8}$. 48-49.

Hellwig, 8. 7-8.

KAPITEL III

1

2

Kutscher, 1964, 8. 106. ebd., s. 108. 3

Schudraka, Vasantasena, trans. Hertha und Kurt Martens (Starnberg am See, 1947), 8. 68.

4

Frank Wedekind, Frank Wedekind: Prosa, Dramen, Verse, B. 2 (Milnchen, 1969), 8. 93.

5

ebd., 8.93.

6

Joachim Friedenthal, "Nachwort", Frank Wedekind: Gesammelte Werke, B. 9 (Mllnchen, 1924), 8. 461-462.

Rothe, 8. 53-54.

8

Frank Wedekind, "Das Sonnenspektrum", Frank Wedekind:

Prosa, Dramen, Verse, B. 2 (Mlinchen, 1969), s. 94.

Ernst Schwelzer, Das Groteske und das Drama Frank Wedekinds $_{10}$ (Diss. Tubingen, 1932), s. 73.

11

Wedekind, $1969,8.94$.

12

ebd., 8. 95.

13

ebd., 8. 95-96.

14

ebd., s. 97.

15

ebd., s. 104.

16

ebd., 8. 105.

17

ebd., 8. 105.

18

ebd., s. 107.

19

ebd., 8. 107.

ebd., 8. 108-109. 
20

21

22

23

24

ebd., 8. 109.

ebd., 8. 115.

ebd., 8. 118 .

ebd., 8. 118.

25

ebd., s. 104.

Richard von Krafft-Ebing, Psychopathia Sexualis (Stuttgart, 1888$),$ s. 151 .

Wedekind, 1969, s. 121.

27

28

ebd., s. 123.
ebd., 8.124.

29. 30

ebd., 8. 124.

31

Rothe, 8.54. 32

Wedekind, 1969, 8. 119.

33

ebd., 8. 130 .

Rothe, 8.53.

KAPITEL IV

1

Frank Wedekind, "Was ich mir dabei dachte", Frank Wedekind: Prosa, Dramen, Verse, B. 1 (Mltuchen, kein Datum), 8. 949.

2

Frank Wedekind, "Tod und Teufel", Frank Wedekind:

Prosa, Dramen, Verse, B. 2 (Mllnchen, 1969), 8. 207.

Wedekind, kein Datum, s. 950.

4

Dehnow, 8. 91 .

5

Wedekind, "Tod", 8. 208.

6

ebd., 8. 209.

7

ebd., 8. 209-210.

8

ebd., 8. 210.

9

ebd., 8. 210-211. 
10

11

ebd., 8. 212 .

11

12

ebd., 8. 212 .

13

ebd., 8. 213.

14

ebd., 8. 213.

14

15

ebd., 8. 215.

16

ebd., 8.216.

16

17

ebd., 8.219.

18

$19^{\text {ebd., s. } 220 .}$

ebd., 8. 219.

20 ebd., 8. 220 .

ebd., 8. 220.

21

ebd., 8. 221.

22

Wedekind, kein Datum, 8. 950.

23 24

Wedekind, "Tod", 8. 221.

Lujo Bassermann, Das Hlteste Gewerbe: eine Kulturgeschichte (Frankfurt, 1965), 8. 49.

25

26 WedekInd, "Tod", 8. 221.

27

ebd., 8. 222 .

28

ebd., 8. 222 .

29

ebd., 8. 222 .

ebd., 8. 220.

31

ebd., 8. 224 .

32

33

ebd., 8 . 224.

34 ebd., 8. 225.

35

ebd., 8. 227.

ebd., 8. 227-228. 
36

37

ebd., 8. 228.

38

ebd., 8. 228.

39

ebd., s. 229.

ebd., 8.230.

40

41

ebd., 8. 230 .

42

ebd., 8. 231.

43

Schweizer, 8. 72 .

Wedekind, "Tod", 8. 232. 44

Friedenthal, 1914.

\section{KAPITEL V}

1

Wedekind, kein Datum, 8. 968.

2

ebd., 8. 968 .

3

ebd., 8. 968 .

Kutscher, 1964, 8. 290.

5

Siegfried Melchinger, "Frank Wedekind", Welttheater (Braunschweig, 1962), 8. 403 .

6

Frank Wedekind, "Schloss Wetterstein", Frank Wedekind:

Prosa, Dramen, Verse, B. 2 (MUnchen, 1969), 8. 406.

Rothe, 8. 123.

8

Wedekind, "Schloss", 8. 407.

9

10

ebd., 8. 408 .

11

ebd., 8. 416-417.

12

ebd., 8. 422 .

13

14

ebd., 8. 422 .

ebd., 8.423.

Rothe, 8. 124.

15

Wedekind, "Sch1088", 8. 426. 
16

ebd., 8. 427.

17

18

$$
\text { ebd., } 8.427 .
$$

19

ebd., 8. 427.

20

ebd., 8.430 .

21

ebd., 8. 430 .

22

ebd., 8. 432 .

23

ebd., s. 432 .

24

ebd., 8. 433 .

25

ebd., 8 . 433-434.

26

Rothe, 8. 124.

Arthur Kutscher, "Schloss Wetterstein", Frank Wedekind und das Theater, ed. Drei Masken Verlag (Berlin, 1915), 8, 59.

Peter Michelsen, "Frank Wedekind", Deutsche Dichter

der Moderne, ed. Benno von Wiese (Berlin, 1965), B. 58.

\section{KAPITEL VI}

1

Schweizer, 8. 75 .

2

Elise Dosenheimer, Das deutsche soziale Drama von LessIng bis Sternheim (Konstanz, 1949), 8. 187.

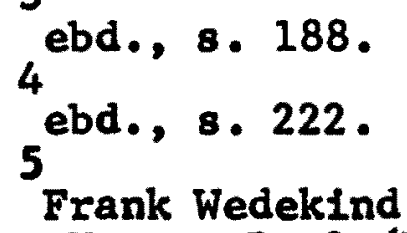

Dramen, Verse, B. 2 (Munchen, 1969), 8. 794 . 
BIBLIOGRAPHIE

Alker, Ernst. Die Deutsche Literatur im 19. Jahrhundert. Stuttgart: Krbner, 1969.

Andreas-Sa lome, Lou. "Frlhlings Erwachen", Die Zukunft. B. 58 (1907).

Bassermann, Lujo. Das Hlteste Gewerbe: eine Kulturgeschichte. Frankfurt: Ulistein, 1965.

Brecht, Bertolt. Schriften zum Theater I: 1918-1933. Frankfurt: Suhrkamp, 1963.

Dehnow, Fritz. Frank Wedekind. Leipzig: 0. R. Reisland, 1922 .

Dietrich, Felix. Internationale Bibliographie der Zeitschriftenilteratur. Osnabrlick: Felix Dletrich, 18981969.

Dosenheimer, Elise. Das deutsche sozlale Drama von Lessing bis Sternheim. Konstanz: Sudverlag, 1949.

Drei Masken Verlag, ed. Frank Wedekind und das Theater. Berlin, 1915 .

Engels, Friedrich. Der Ursprung der Familie, des Privateigentums und des staats. Berlin: Dietz, 1969.

Fechter, Paul. Frank Wedekind: Der Mensch und das Werk. Jena: Erich Lichtenstein, 1920.

-.-.. Geschichte der deutschen Literatur, Bd. 1. Glters loh: Sigbert Mohn, 1960.

Feuchtwanger, Lion. "Frank Wedekind", Neue Deutsche Literatur, 12 J., N. 7 (1964).

Film. "Wedekind wider die Damen", Der Spiegel, 16 J., N. 4, (1962).

Friedentha1, Joachim, ed. Das Wedekindbuch. MUnchen: MUller, 1914.

-Nachwort", Frank Wedekind: Gesammelte Werke, Bd. 9. Mlinchen: Miller, 1924.

Frledrich, Hans. "Bestie Mensch", Frankfurter Hefte, N. 11, (1955). 
Gundolf, Friedrich. Frank Wedekind. MUnchen: MUllerLangen, 1954.

Glinther, Herbert. "Paris als Erlebnis: Frank Wedekind und Paris", Antares, 1 J., Nr. 5 (Juni, 1953).

Heinisch, Klaus J. Der Utopische Staat. Leck: Rowohlt, 1962 .

Hellwig, Hans. "Frank Wedekinds dichterische AnfHnge", Dissertation von Giessen, 1928.

Hensel, Georg. Splelplan: Schauspiel fuhrer von der Antike bis zur Gegenwart, B. II. Ber11n: Propy1den, 1966.

Heuss, Theodor. "Frank Wedekind", Der Kunstwart, XXII, 17, 1. Juniheft (1909).

Hof fmann-Krayer und Hanns BHchtold-StHubl1, eds. Handw Urterbuch des Deutschen Aberglaubens, 10 Bde. BerIIn und Lelpzig: Walter de Gruyter, 1927-1942.

Holl, Karl. Geschichte des deutschen Lustsplels. Leipzig: Weber, 1923.

Kerr, Hans. Frank Wedekind als Mensch und Kllnstler. Berlin: Hermann Walther, 1909.

Kessel, Martin. "Frank Wedekind", Aufbau, 4. J., Heft 3 (1948).

Kosch, Wilhelm. Deutsches Literatur-Lexikon, Bd. 4. Bern: A. Francke, 1958.

Krafft-Ebing, Richard von. Psychopathia Sexualis. Stuttgart: Enke, 1888 .

Kutscher, Arthur. "Frank Wedekinds Stoffe und Sti1", Welt und Wort, 19 J., H. 7 (1964).

1964.

Wedekind: Leben und Werk. MUnchen: List,

Lion, Ferdinand. 'Wedekind redivivus?", Merkur, 16 J.' (1962).

Mann, Otto. Geschichte des deutschen Dramas. Stuttgart: Kroner, 1960.

-.......-. Deutsche Literaturgeschichte. Glters1oh: Bertelsmann, 1964.

Martini, Fritz. Deutsche Literaturgeschichte. Stuttgart: 
Kr ४ner, 1968.

Marx, Karl und Friedrich Engels. Manifest der Kommunistischen Partel. Peking: Verlag flur Fremdsprachige LIteratur, 1969.

Melchinger, Siegfried und Henning Rischbieter, eds. "Frank Wedekind" von Siegried Melchinger, Welttheater. Braunschweig: Georg Westermann, 1962.

Nadler, Josef. Geschichte der deutschen Literatur. Regensburg: Habbel, 1961.

Naumann, Hans. Die deutsche Dichtung der Gegemwart: 18851933. Stuttgart: Metzlerscher, 1933.

Reich, Willi, ed. Frank Wedekind:X Selbstdarstellung. Munchen : Langen-Muller, 1954.

Rilla, Paul. "Auseinandersetzung mit Wedekind", Vom burger1ichen zum sozialistischen Realismus. Leipzig: Philipp Reclam Jun., 1967.

Rothe, Friedrich. Frank Wedekinds Dramen: Jugendstil und Lebensphilosophie. Stuttgart: J. B. Metzlersche, 1968.

Schaukal, Richard von. "Frank Wedekind: Skizze zu einem PortrHt", Uber Dichter. Mitnchen: Langen-MUller, 1966.

Schlegel, Friedrich. Lucinde. Stuttgart: PhIlipp Reclam Jun., 1964.

Schneider, Georg. "Frank Wedekind und seine Modelle", Welt und Wort, 9 J. (1954).

Schudraka. Vasantasena, Ubersetzung von Hertha und Kurt Martens. Starnberg am See: Bachmair, 1947.

Schultze, Friedrich, ed. Theater im Gespritch: Ein Forum der Dramaturgie. Munchen: Langen-MUIler, 1963.

Schweizer, Ernst. "Das Groteske und das Drama Frank Wedekinds", Dissertation von Tlbingen, 1932 .

Seehaus, Glinter. Frank Wedekind und das Theater. MUlnchen: Laokoon, 1964.

Sokel, Walter H. The Writer in Extremis. Stanford: Stanford University Press, 1959.

Stadtbibliothek MUnchen, ed, Frank Wedekind: zum 100. Geburtstag. Munchen, 1964 . 
Steffen, Hans, ed. "Die kombdiantischen Grotesken Frank Wedekinds" von Paul B Hckmann, Das deutsche Lustsplel II. Gbttingen: Vandenhoeck und Ruprecht, 1969.

Vieweger, Erich. Frank Wedekind und sein Werk. Chemnitz: Selbstverlag, 1919.

v81ker, Klaus. Wedekind. Velber bei Hannover: Friedrich, 1965.

Wedekind, Frank. Gesammelte Werke, 9 Bde. Mllnchen: Miller, 1924.

\section{4.}

Gesammelte Briefe, 2 Bde. Munchen: Mtllier,

"Was aber Irgend Ubrig...", Welt und Wort,

19 J., H. 7. (1964).

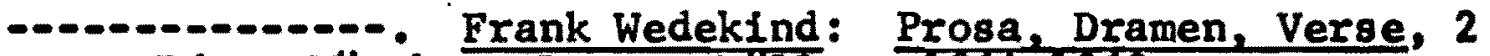

Bde. Minchen: Langen-Mliler, 1964-1969.

-.-.-.-.-. Der Vermumte Herr: Briefe Frank Wedekinds aus den Jahren 1881-1917. Minchen: Deutscher Taschenbuch, 1967.

Wedekind, Tilly. Lulu-die Rolle meines Lebens. Minchen: RUtten und Loening, 1969.

Wiese, Benno von, ed. "Frank Wedekind" von Peter Michelsen, Deutsche Dichter der Moderne. Berlin: Erich Schmidt, 1965.

W1lpert, Gero von. Deutsches Dichterlexikon. Stuttgart: Kryner, 1963. 